\title{
Closure Theorems for Orientor Fields and Weak Convergence
}

\author{
LAMBerto CesARI
}

\author{
Communicated by J. SERRIN
}

\section{Introduction}

In the theory of optimization, in connection with ordinary and partial differential equations, a number of closure and lower closure theorems have been obtained in different contexts and under a variety of conditions and modes of convergence. In particular "seminormality" conditions (property $(Q)$ and its variants) have played different roles. In this paper we first prove closure and lower closure theorems for orientor fields in a rather abstract context, all based on weak convergence and MAZUR's theorem ( $\$ 4$ and 5). In the context of orientor fields, these theorems can be given the most satisfactory formulation and simplest proofs (see, e.g., th. (4.i), (5.i)). Furthermore, in the present new approach, the interplay of "seminormality" conditions and modes of convergence can be easily seen: the stronger the mode of convergence, the weaker are the "seminormality" conditions that are needed. From these theorems we then derive, as corollaries, closure and lower closure statements for Mayer and Lagrange problems ( $\$ 6$ and 7) and lower semicontinuity statements for free problems (\$8). Under suitable hypotheses, no seminormality condition (or property $(Q)$ ) is needed. Further theorems without seminormality conditions, as well as other details, are discussed in [3]. In particular, we show that seminormality conditions can be removed, not only under standard Lipschitz requirements, as expected, but also under much more satisfactory simple growth conditions, as proposed some time ago by E. H. Rothe for free problems. Applications to multidimensional Lagrange problems are discussed in [8].

\section{Notations}

We shall denote by $\operatorname{cl} Z$ and $\operatorname{co} Z$ the closure and the convex hull of any arbitrary subset $Z$ of $E_{N}$, and we denote by $|Z|$ its Lebesgue measure if $Z$ is measurable.

Let $A$ denote any set of the $t x$-space $E_{v} \times E_{n}, t=\left(t^{1}, \ldots, t^{\nu}\right), x=\left(x^{1}, \ldots, x^{n}\right)$; for any $t \in E_{v}$ let $A(t)=\left[x \in E_{n} \mid(t, x) \in A\right]$ and let $A_{0}$ denote the set of all $t$ for which $A(t) \neq \emptyset\left(A_{0}\right.$ is the projection of $A$ on $\left.E_{v}\right)$. Let $G$ be any measurable subset of $E_{v}$ with finite measure, $G \subset A_{0} \subset E_{v}$. For every $(t, x) \in A$ let $Q(t, x)$ be a given subset of the $z$-space $E_{r}, z=\left(z^{1}, \ldots, z^{r}\right)$. 
We shall consider pairs $\xi(t)=\left(\xi^{1}, \ldots, \xi^{r}\right), x(t)=\left(x^{1}, \ldots, x^{n}\right), t \in G$, of measurable functions on $G$ such that

$$
\xi(t) \in Q(t, x(t)), \quad t \in G \quad \text { (a.e.). }
$$

We call (2.1) an abstract orientor field equation. In most applications, of course, $\xi$ is actually related to $x$ by some differential or more general relation. We shall mention this situation whenever it is relevant.

For every $(\bar{t}, \bar{x}) \in A$ and $\delta>0$ we denote by $N_{\delta}(\bar{t}, \bar{x})\left[N_{\delta}, \bar{t}(\bar{x})\right]$ the set of all points $(t, x) \in A[(\bar{t}, x) \in A]$ at a distance $\leqq \delta$ from $(\bar{t}, \bar{x})$. The following concepts of upper semicontinuity of variable sets are of interest here. We say that the sets $Q(t, x)$ satisfy property $(K)$ with respect to $(t, x)$ at a point $(\bar{t}, \bar{x}) \in A$ (i.e. Kuratowsky's upper semicontinuity condition), provided

$$
Q(\bar{t}, \bar{x})=\bigcap_{\delta>0} \mathrm{cl} \bigcup_{(t, x) \in N_{\delta}(\bar{t}, \bar{x})} Q(t, x) .
$$

We say that the sets $Q(t, x)$ satisfy property $(Q)$ with respect to $(t, x)$ at a point $(\bar{t}, \bar{x}) \in A$, provided

$$
Q(\bar{t}, \bar{x})=\bigcap_{\delta>0} \operatorname{cilco} \bigcup_{(t, x) \in N_{\delta}(\bar{t}, \bar{x})} Q(t, x) .
$$

We say that the sets $Q(t, x)$ satisfy property $(K)$, or $(Q)$, with respect to $x$ only at $(\bar{t}, \bar{x})$ provided relation $(2.2)$, or $(2.3)$, holds with $N_{\delta, \bar{t}}(\bar{x})$ replacing $N_{\delta}(\bar{t}, \bar{x})$. We shall say that the sets $Q(t, x)$ satisfy property $(K)$, or property $(Q)$, with respect to $(t, x)$, or with respect to $x$ only, in a subset $A^{\prime}$ of $A$, if the corresponding properties hold at every $(t, x) \in A^{\prime}$.

Sets $Q(t, x)$ possessing property $(K)$ are closed since they are intersections of closed sets; sets possessing property $(Q)$ are closed and convex since they are intersections of closed and convex sets.

We introduced property $(Q)$ in [1 a], where we also proved a number of statements relating properties $(K)$ and $(Q)$ (property $(K)$ was called property $(U)$ in [1 a]). In [1 bcd] we stated a number of criteria in order that the sets which are relevant in Lagrange problems have property $(Q)$. For equibounded compact sets property $(K)$ reduces to the usual metric upper semicontinuity of sets; for equibounded compact convex sets property $(Q)$ also reduces to metric upper semicontinuity.

In [1 cd] we proved that property $(Q)$ for Lagrange problems is the extension of TONELli's and McSHANE's seminormality condition for free problems.

\section{Closure Theorems with respect to Uniform Convergence and Variants}

These theorems have been proved in earlier papers [1 ab] for the case $v=1$, $r=n, G=[a, b]$, a fixed interval in $E_{1}, x$ absolutely continuous $(\mathrm{AC})$, and $\xi(t)=$ $x^{\prime}(t), t \in[a, b]$ (a.e.).

(3.i) (L. CESARI [1 a]). If $A$ is closed and contained in $E_{1} \times E_{n}$, if $T_{0}$ is a subset of $A_{0}$ of measure zero, $T_{0} \subset A_{0} \subset E_{1}$, with $G=[a, b] \subset A_{0}, H_{0}=A \cap\left(T_{0} \times E_{n}\right)$, $A_{G}=A \cap\left(G \times E_{n}\right)$, if the sets $Q(t, x)$ satisfy property $(Q)$ with respect to $(t, x)$ in $A_{G}-H_{0}$, if $x(t), x_{k}(t), a \leqq t \leqq b$, are absolutely continuous, if

$$
\left(t, x_{k}(t)\right) \in A, \quad t \in[a, b], \quad x_{k}^{\prime}(t) \in Q\left(t, x_{k}(t)\right), \quad t \in[a, b] \text { (a.e.), }
$$


$k=1,2, \ldots$, and if $x_{k}(t) \rightarrow x(t)$ uniformly in $[a, b]$ as $k \rightarrow \infty$, then

$$
(t, x(t)) \in A, \quad t \in[a, b], \quad x^{\prime}(t) \in Q(t, x(t)), \quad t \in[a, b] \text { (a.e.). }
$$

Remark 1. We have shown in ([1 e], p. 313) by means of an example that property $(Q)$ " with respect to $(t, x)$ " is essential in (3.i). In the example in [1 e], $A=[a, b] \times[c, d], \nu=r=n=1$, and there is a partition $[a, b]=S_{1} \cup S_{2}$, with $S_{1} \cap S_{2}=\emptyset, S_{1}$ open, $S_{2}$ closed, both $S_{1}$ and $S_{2}$ of positive measure, the sets $Q(t)$ independent of $x$, such that, if $A_{i}=S_{i} \times[c, d], i=1,2$, then the sets $Q(t)$ satisfy property $(Q)$ both in $A_{1}$ and in $A_{2}$ (but not in $A$ ), and the contention of (3.i) is not true. Thus in (3.i) property $(Q)$ "with respect to $x$ only" would not suffice. Also note that (in view of $\S 4$ below) in (3.i) the uniform convergence $x_{k}(t) \rightarrow x(t)$ does not imply weak convergence of the derivatives $x_{k}^{\prime}$ toward $x^{\prime}$ in $L_{1}$, as the following trivial example shows: $v=r=n=1, x(t)=0, x_{k}(t)=k^{-1} \sin k^{2} t$, $0 \leqq t \leqq 2 \pi, k=1,2, \ldots$, where the derivatives $x_{k}^{\prime}(t)=k \cos k^{2} t$ are not bounded in the $L_{1}$-norm and certainly do not converge weakly to zero.

\section{Closure Theorems for Orientor Fields with Respect to Weak Convergence of the Derivatives}

We shall use the same notations as in $\S 2$. Thus points in the $E_{v}, E_{n}, E_{r}$ spaces will be denoted by $t=\left(t^{1}, \ldots, t^{v}\right), x=\left(x^{1}, \ldots, x^{n}\right), \xi=\left(\xi^{1}, \ldots, \xi^{r}\right)$. Also, $A$ is a subset of $E_{v} \times E_{n}, A_{0}$ the projection of $A$ on $E_{v}$, and $A(t)=\left[x \in E_{n} \mid(t, x) \in A\right]$. For every $(t, x) \in A$ a subset $Q(t, x)$ of $E_{r}$ is assigned. We shall denote by $G$ and $T_{0}$ given subsets of $A_{0}$ in $E_{v}, T_{0} \subset G$; then $A_{G}$ and $H_{0}$ are the sets $A_{G}=A \cap\left(G \times E_{n}\right)$ and $H_{0}=A \cap\left(T_{0} \times E_{n}\right)$. We shall finally denote by $x(t)=\left(x^{1}, \ldots, x^{n}\right)$ and $\xi(t)=$ $\left(\xi^{1}, \ldots, \xi\right), t \in G$, given functions on $G$.

(4.i) If $G$ is a measurable set with finite measure and $T_{0}$ has measure zero, if the sets $A(t)$ are closed for $t \in G-T_{0}$, if the sets $Q(t, x)$ satisfy property $(Q)$ with respect to $x$ in $A_{G}-H_{0}$, if $\xi(t), x(t), \xi_{k}(t), x_{k}(t), t \in G, k=1,2, \ldots$, are measurable functions, $\xi, \xi_{k} \in\left(L_{1}(G)\right)^{r}$, if

$$
x_{k}(t) \in A(t), \quad \xi_{k}(t) \in Q\left(t, x_{k}(t)\right), \quad t \in G \text { (a.e.), } k=1,2, \ldots,
$$

and if $\xi_{k} \rightarrow \xi$ weakly in $\left(L_{1}(G)\right)^{r}$ and $x_{k}(t) \rightarrow x(t)$ in measure in $G$ as $k \rightarrow \infty$, then

$$
x(t) \in A(t), \quad \xi(t) \in Q(t, x(t)), \quad t \in G \text { (a.e.). }
$$

Proof. By extraction of a suitable subsquence we may assume that $x_{k}(t) \rightarrow x(t)$ pointwise a.e. in $G$. Let $T_{0}^{\prime}$ be the subset of measure zero of all $t \in G$ where $x_{k}(t)$ does not converge or does not converge to $x(t)$, or where $x(t)$ is not finite. Then $x(t) \in A(t)$ for all $t \in G-\left(T_{0} \cup T_{0}^{\prime}\right)$. For every $s=1,2, \ldots$, let us consider the sequence $\left[\xi_{s+k}(t), t \in G, k=1,2, \ldots\right]$. By virtue of the Banach-Saks-Mazur theorem (see S. MAZur [9], or M. DAY [5], p. 46, or K. Yosida [11], p. 120), there is a set of real numbers $c_{N k}^{(s)} \geqq 0, k=1,2, \ldots, N, N=1,2, \ldots$, with $\sum_{k=1}^{N} c_{N k}^{(s)}=1$, such
that if

$$
\xi_{N}^{(s)}(t)=\sum_{k=1}^{N} c_{N k}^{(s)} \xi_{s+k}(t), \quad t \in G, \quad N=1,2, \ldots
$$


then $\xi_{N}^{(s)}(t) \rightarrow \xi(t)$ strongly in $\left(L_{1}(G)\right)^{r}$ (that is, in the $L_{1}$-norm in $G$ ), this being true for every $s=1,2, \ldots$. Then for every $s$ there is also a subset $T_{s}$ of measure zero of points in $G$, and a sequence of integers $N_{\lambda}^{(s)}, \lambda=1,2, \ldots$, with $N_{\lambda}^{(s)} \rightarrow \infty$, such that, for $t \in G-T_{s}, \xi(t)$ is finite and $\xi_{N}^{(s)} \rightarrow \xi(t)$ as $\lambda \rightarrow \infty$. Let $T$ denote the subset of measure zero in $G$ which is the union of all sets $T_{0}, T_{0}^{\prime}, T_{s}, s=1,2, \ldots$

Now let $t_{0}$ be any point in $G-T$, and take $x_{0}=x\left(t_{0}\right)$. Then $\left(t_{0}, x_{k}\left(t_{0}\right)\right) \rightarrow$ $\left(t_{0}, x_{0}\right) \in A$, the sets $Q(t, x)$ have property $(Q)$ with respect to $x$ at $\left(t_{0}, x_{0}\right)$, and, given $\varepsilon>0$, there is some $s_{0}$ such that $\left|x_{s}\left(t_{0}\right)-x_{0}\right| \leqq \varepsilon$ for all $s \geqq s_{0}$. For $s \geqq s_{0}$ we have then

$$
\xi_{s+k}\left(t_{0}\right) \in Q\left(t_{0}, x_{s+k}\left(t_{0}\right)\right), \quad\left|x_{s+k}\left(t_{0}\right)-x_{0}\right| \leqq \varepsilon, \quad k=1,2, \ldots,
$$

and hence

$$
\sum_{k=1}^{N} c_{N k}^{(s)} \xi_{s+k}\left(t_{0}\right) \in \mathrm{co} \underset{x \in N_{\varepsilon, t_{0}}\left(x_{0}\right)}{\bigcup} Q\left(t_{0}, x\right) .
$$

Finally, for $N=N_{\lambda}$ and $\lambda \rightarrow \infty$, we have

$$
\xi\left(t_{0}\right) \in \mathrm{cl} \operatorname{co} \underset{x \in N_{\varepsilon, t_{0}}\left(x_{0}\right)}{\bigcup} Q\left(t_{0}, x\right),
$$

where $\varepsilon>0$ is arbitrary. By property $(Q)$ with respect to $x$ only at $\left(t_{0}, x_{0}\right)$ we have $\xi\left(t_{0}\right) \in Q\left(t_{0}, x_{0}\right)$. We have proved that $\xi(t) \in Q(t, x(t))$ for all $t \in G-T$, that is, a.e. in $G$. Statement (4.i) is thereby proved.

Note that in the proof of (4.i) we do not need the full force of property $(Q)$ with respect to $x$. All we need is that there is a subsequence $k_{s}$ such that (i) $x_{k_{s}}(t) \rightarrow x(t)$ pointwise a.e. in $G$ and (ii) for almost all $t \in G$ we have

$$
Q(t, x(t)) \supset \bigcap_{h=1}^{\infty} \mathrm{cl} \mathrm{co}\left\{\bigcup_{s=h}^{\infty} \zeta_{k_{s}}(t)\right\} .
$$

The same remark holds for theorem (4.ii) below.

Remark 2. In the situation of $\S 3$, with $v=1, r=n, G=[a, b]$, a fixed interval in $E_{1}, x, x_{k}$ absolutely continuous, and $\xi(t)=x^{\prime}(t), \xi_{k}(t)=x_{k}^{\prime}(t), t \in[a, b]$ (a.e.), then the weak convergence $\xi_{k} \rightarrow \xi$ (that is, $x_{k}^{\prime} \rightarrow x^{\prime}$ weakly in $L_{1}([a, b])$ ) required in (4.i) together with the convergence $x_{k}(\bar{t}) \rightarrow x(\bar{t})$ at least at one point $\bar{t}$, certainly implies the uniform convergence $x_{k} \rightarrow x$ in $[a, b]$. Indeed for every $t \in[a, b]$ we have $x_{k}(t)=x_{k}(\bar{t})+\int_{\bar{t}}^{t} x_{k}^{\prime}(\tau) d \tau$, and the weak convergence $x_{k}^{\prime} \rightarrow x^{\prime}$ in $L_{1}$ implies the pointwise convergence of $x_{k}(t)$ at every $t$. On the other hand, by a theorem of Dunford-PetTIs [6] (see also R. E. Edwards [7], p. 274) the functions $x_{k}^{\prime}$ are equiabsolutely integrable in $[a, b]$; hence the functions $x_{k}$ are equiabsolutely continuous in $[a, b]$ and the convergence $x_{k} \rightarrow x$ is therefore uniform. In this situation, if we have $A$ closed, property $(Q)$ with respect to $x$ in $A-H_{0},\left(t, x_{k}(t)\right) \in A$, $t \in[a, b], x_{k}^{\prime}(t) \in Q\left(t, x_{k}(t)\right), t \in[a, b]$ (a.e.), and $x_{k}^{\prime} \rightarrow x^{\prime}$ weakly in $\left(L_{1}(G)\right)^{n}$ as $k \rightarrow \infty$, then certainly $(t, x(t)) \in A, t \in[a, b]$, and $x^{\prime}(t) \in Q(t, x(t)), t \in[a, b]$ (a.e.).

(4.ii) Let the hypotheses of (4.i) hold, except that the sets $Q(t, x)$ satisfy only property $(K)$ with respect to $x$ in $A_{G}-H_{0}$, the functions $\xi, x, \xi_{k}, x_{k}$ are only measurable in $G$, and $\xi_{k}(t) \rightarrow \xi(t), x_{k}(t) \rightarrow x(t)$ pointwise a.e. in $G$. Then the conclusion of (4.i) also holds. 
Proof. Let $T_{0}^{\prime}$ be the subset of measure zero of all $t \in G$ where $x_{k}(t)$ does not converge to $x(t)$, or $\xi_{k}(t)$ does not converge to $\xi(t)$. Since $A$ is closed we have $(t, x(t)) \in A$ a.e. in $G$, as in the proof of (4.i). The set $T=T_{0} \cup T_{0}^{\prime}$ also has measure zero in $G$. For every $t_{0} \in G-T$ put $x_{0}=x\left(t_{0}\right)$, and note that, given $\varepsilon>0$, there is $k_{0}>0$ such that $\left|x_{k}\left(t_{0}\right)-x_{0}\right| \leqq \varepsilon$ for all $k \geqq k_{0}$. Then, for $k \geqq k_{0}$, from $\xi_{k}\left(t_{0}\right) \in$ $Q\left(t_{0}, x_{k}\left(t_{0}\right)\right)$ we derive

Finally, as $k \rightarrow \infty$,

$$
\xi_{k}\left(t_{0}\right) \in \bigcup_{x \in N_{\varepsilon, t_{0}}\left(x_{0}\right)} Q\left(t_{0}, x\right), \quad k \geqq k_{0} .
$$

$$
\xi\left(t_{0}\right) \in \mathrm{cl} \underset{x \in N_{\varepsilon, t_{0}}\left(x_{0}\right)}{\bigcup} Q\left(t_{0}, x\right) .
$$

This relation holds for all $\varepsilon>0$; hence by property $(K)$ at $\left(t_{0}, x_{0}\right)$ with respect to $x$ only, we have

$$
\xi\left(t_{0}\right) \in \bigcap_{\varepsilon>0} \mathrm{cl} \bigcup_{x \in N_{\varepsilon, t_{0}}\left(x_{0}\right)} Q\left(t_{0}, x\right)=Q\left(t_{0}, x_{0}\right) .
$$

We have proved that $\xi(t) \in Q(t, x(t))$ a.e. in $G$.

Remark 3. Theorem (4.iii) below concerns the case where the pairs $\xi_{k}(t)$, $x_{k}(t), t \in G$, satisfying $\xi_{k}(t) \in Q\left(t, x_{k}(t)\right), t \in G$ (a.e.), $k=1,2, \ldots$, with $\xi_{k} \rightarrow \xi$ weakly in $\left(L_{1}(G)\right)^{r}$, and $x_{k}(t) \rightarrow x(t)$ pointwise a.e. in $G$, can be replaced by modified pairs $\bar{\xi}_{k}(t), x(t), t \in G$, satisfying $\bar{\xi}_{k}(t) \in Q(t, x(t)), t \in G$ (a.e.), $k=1,2, \ldots$, where $\bar{\xi}_{k}$ still converges to $\xi$ weakly in $\left(L_{1}(G)\right)^{r}$ as $k \rightarrow \infty$ and thus $\delta_{k}(t)=\xi_{k}(t)-\bar{\xi}_{k}(t) \rightarrow 0$ weakly in $\left(L_{1}(G)\right)^{r}$ as $k \rightarrow \infty$. This situation actually occurs in a large class of problems (see Remark 8 in $\S 6$ below); in this situation the sets $Q(t, x)$ need be assumed only convex and closed (the properties $(Q)$ or $(K)$ are not required).

(4.iii) Let the hypotheses of (4.i) hold, except that the sets $Q(t, x)$ are assumed to be only closed and convex for every $(t, x) \in A_{G}-H_{0}$, the functions $\xi, \xi_{k} \in\left(L_{1}(G)\right)^{r}$, $x$ is only measurable, $x_{k}(t)=x(t)$ for all $t$ and $k$, and $\xi_{k} \rightarrow \xi$ weakly in $\left(L_{1}(G)\right)^{r}$. Then the conclusion of (4.i) also holds.

Proof. Let $T_{1}$ be the subset of measure zero in $G$ defined in the proof of (4.i) relative to the weak convergence of $\xi_{k}$ to $\xi$ for $s=1$. Then the set $T=T_{0} \cup T_{1}$ also has measure zero in $G$. For every $t_{0} \in G-T$ let $x_{0}=x\left(t_{0}\right)$, and note that $\xi_{1+k}\left(t_{0}\right) \in Q\left(t_{0}, x_{0}\right), k=1,2, \ldots$, where the set $Q\left(t_{0}, x_{0}\right)$ is convex. Hence by using the numbers $c_{N k}^{(1)} \geqq 0, k=1, \ldots, N$, defined in the proof of (4.i) we have

$$
\sum_{k=1}^{N} c_{N k}^{(1)} \xi_{1+k}\left(t_{0}\right) \in Q\left(t_{0}, x_{0}\right) \quad \text { for all } N .
$$

For $N=N_{\lambda}^{(1)}, \lambda \rightarrow \infty$, the first member of this relation approaches $\xi\left(t_{0}\right)$. Since $Q\left(t_{0}, x_{0}\right)$ is closed, we have $\xi\left(t_{0}\right) \in Q\left(t_{0}, x_{0}\right)$. We have proved that $\xi(t) \in Q(t, x(t))$ a.e. in $G$.

Remark 4. D. E. Cowles [4] introduced properties $\left(Q_{\rho}\right)$ intermediate between property $(Q)$ and property $(K)$. These intermediate properties can also be used in connection with the closure and lower closure theorems of the present paper. as P. KAISER will show in [8]. 


\section{Lower Closure Theorems for Orientor Fields with Respect to Weak Convergence of the Derivatives}

We shall use essentially the same notation as in $\$ 2$ and in $\$ 4$. Thus points in $E_{v}, E_{n}, E_{r+1}$ spaces will be denoted by $t=\left(t^{1}, \ldots, t^{v}\right), x=\left(x^{1}, \ldots, x^{n}\right),\left(z^{0}, z\right)=$ $\left(z^{0}, z^{1}, \ldots, z^{r}\right)$, or $(\eta, \xi)=\left(\eta, \xi^{1}, \ldots, \xi\right)$, respectively. Also as before $A$ is a subset of $E_{v} \times E_{n}, A_{0}$ is the projection of $A$ on $E_{v}$, and $A(t)=\left[x \in E_{n} \mid(t, x) \in A\right]$. A subset $\tilde{Q}(t, x)$ of $E_{r+1}$ is assigned for every $(t, x) \in A$. Again we shall denote by $G$ and $T_{0}$ given subsets of $A_{0}$ in $E_{v}$; then $A_{G}=A \cap\left(G \times E_{n}\right)$ and $H_{0}=A \cap\left(T_{0} \times E_{n}\right)$. We shall denote by $x(t)=\left(x^{1}, \ldots, x^{n}\right)$ and $(\eta(t), \xi(t))=\left(\eta, \xi^{1}, \ldots, \xi^{r}\right), t \in G$, given functions on $G$.

(5.i) If $G$ is a measurable set with finite measure, and $T_{0}$ has measure zero, if the sets $A(t)$ are closed for $t \in G-T_{0}$, if the sets $\tilde{Q}(t, x)$ satisfy property $(Q)$ with respect to $x$ in $A_{G}-H_{0}$, and if $\xi(t), x(t), \eta_{k}(t), \xi_{k}(t), x_{k}(t), \lambda(t), \lambda_{k}(t), t \in G$, $k=1,2, \ldots$, are measurable functions, $\xi, \xi_{k} \in\left(L_{1}(G)\right)^{r}, \eta_{k} \in L_{1}(G)$, such that

$$
\begin{gathered}
x_{k}(t) \in A(t), \quad\left(\eta_{k}(t), \xi_{k}(t)\right) \in \tilde{Q}\left(t, x_{k}(t)\right), \quad t \in G(\text { a.e. }), \quad k=1,2, \ldots, \\
-\infty<i=\varliminf_{k \rightarrow \infty} \int_{G} \eta_{k}(t) d t<\infty \\
\xi_{k} \rightarrow \xi \text { weakly in }\left(L_{1}(G)\right)^{r}, \quad x_{k}(t) \rightarrow x(t) \text { in measure in } G \text { as } k \rightarrow \infty, \text { and } \\
\eta_{k}(t) \geqq \lambda_{k}(t), \quad \lambda, \lambda_{k} \in L_{1}(G), \quad \lambda_{k} \rightarrow \lambda \text { weakly in } L_{1}(G)
\end{gathered}
$$

then there is a function $\eta(t), t \in G, \eta \in L_{1}(G)$, such that

$$
x(t) \in A(t), \quad(\eta(t), \xi(t)) \in \tilde{Q}(t, x(t)), \quad t \in G(\text { a.e. }) \quad \text { and } \int_{G} \eta(t) d t \leqq i .
$$

In this formulation of (5.i), $\lambda_{k} \rightarrow \lambda$ weakly in $L_{1}(G)$; hence $\left\|\lambda_{k}\right\|_{1}$ is a bounded sequence, and the part $i>-\infty$ of requirement (5.2) is a consequence of (5.4).

Remark 5. Lower closure theorems are usually applied in situations where it is well known that

$$
i=\varliminf_{k \rightarrow \infty} \int_{G} \eta_{k}(t) d t<\infty .
$$

Functions $\lambda, \lambda_{k}$ satisfying (5.4) of statement (5.i) are easily found (consequently $i>-\infty$ ) if we know, for instance, that one of the following conditions is verified:

$(\alpha)$ There is a real valued function $\psi(t) \geqq 0, t \in G, \psi \in L_{1}(G)$, such that we have $z^{0} \geqq-\psi(t)$ for every $(t, x) \in A_{G}$ and $\left(z^{0}, z\right) \in \tilde{Q}(t, x)$.

Indeed, we have then $\eta_{k}(t) \geqq \lambda_{k}(t)=-\psi(t), t \in G, k=1,2, \ldots$, and $\lambda=-\psi$.

$(\beta)$ There is a real valued function $\psi(t) \geqq 0, t \in G, \psi \in L_{1}(G)$, and a constant $\gamma \geqq 0$ such that for all $(t, x) \in A_{G}$ and $\left(z^{0}, z\right) \in \tilde{Q}(t, x)$ we have $z^{0} \geqq-\psi(t)-\gamma|z|$.

Indeed, we have then $\eta_{k}(t) \geqq \lambda_{k}(t)=-\psi(t)-\gamma\left|\xi_{k}(t)\right|, t \in G, k=1,2, \ldots$. Since $\xi_{k} \rightarrow \xi$ weakly in $\left(L_{1}(G)\right)^{r}$ by hypothesis, by the Dunford-Pettis theorem the functions $\xi_{k}$ are equiabsolutely the integrable in $G$. Hence by the same theorem the sequence $\left|\xi_{k}(t)\right|, t \in G, k=1,2, \ldots$, is weakly compact in $L_{1}(G)$, and there is, therefore, a subsequence $\left[k_{s}\right]$ such that $\lambda_{k_{s}}(t)=-\psi(t)-\gamma\left|\xi_{k_{s}}(t)\right|, t \in G, k=1,2, \ldots$, 
is weakly convergent in $L_{1}(G)$ toward some function $-\psi(t)-\gamma \sigma(t), \sigma(t) \geqq 0, t \in G$. We can thus apply (5.i).

$(\gamma)$ The functions $x$ and $x_{k}$ are in $\left(L_{p}(G)\right)^{n},\left\|x_{k}-x\right\|_{p} \rightarrow 0$ as $k \rightarrow \infty$ for some $p$, $1 \leqq p<\infty$, and there is a real valued function $\psi(t) \geqq 0, t \in G, \psi \in L_{1}(G)$, and a pair of constants $\gamma, \gamma^{\prime} \geqq 0$ such that for all $(t, x) \in A_{G}$ and $\left(z^{0}, z\right) \in \tilde{Q}(t, x)$ we have $z^{0}>-\psi(t)-\gamma^{\prime}|x|^{p}-\gamma|z|$.

The argument is similar to the one above, since $\left\|x_{k}-x\right\|_{p} \rightarrow 0$ implies

$$
\left\|\left|x_{k}\right|^{p}-|x|^{p}\right\|_{1} \rightarrow 0
$$

thus the sequence $\lambda_{k}(t)=-\psi(t)-\gamma^{\prime}\left|x_{k}(t)\right|^{p}-\gamma\left|\xi_{k}(t)\right|, t \in G, k=1,2, \ldots$, certainly possesses a weakly convergent subsequence in $L_{1}(G)$. Instead of the requirement $x, x_{k} \in\left(L_{p}(G)\right)^{n},\left\|x_{k}-x\right\|_{p} \rightarrow 0$, we may require $x_{k}^{i}, x^{i} \in L_{p_{i}}(G),\left\|x_{k}^{i}-x^{i}\right\|_{p_{i}} \rightarrow 0$ for different $p_{i}, 1 \leqq p_{i}<\infty, i=1, \ldots, n$. This remark holds throughout this and the following sections.

$(\delta)$ The functions $x_{k}$ are in $\left(L_{\infty}(G)\right)^{n},\left\|x_{k}\right\|_{\infty} \leqq L_{0}, \xi_{k} \in\left(L_{\infty}(G)\right)^{r},\left\|\xi_{k}\right\|_{\infty} \leqq L_{1}$ for given constants $L_{0}, L_{1}$, and there exists a real valued function $\psi(t) \geqq 0, t \in G$, $\psi \in L_{1}(G)$, and a real valued monotone nondecreasing function $\sigma(\xi), 0 \leqq \xi<+\infty$, such that for all $(t, x) \in A$ and $\left(z^{0}, z\right) \in \tilde{Q}(t, x)$ we have $z^{0} \geqq-\psi(t) \sigma(|x|+|z|)$.

The argument is similar to the one above.

In Remark 5 below we shall show by means of an example that conclusion (5.5) may not hold without requirement (5.4)

Proof of (5.i). Let $j_{k}=\int_{G} \eta_{k}(t) d t, k=1,2, \ldots$ By taking a suitable subsequence we may assume that $j_{k} \rightarrow i$ and $x_{k}(t) \rightarrow x(t)$ pointwise a.e. in $G$ as $k \rightarrow \infty$. Here $-\infty<i<\infty$, so that, if $\rho_{s}$ denotes the maximum of $\left|j_{k}-i\right|$ for $k \geqq s+1$, we have $\rho_{s} \rightarrow 0$ as $s \rightarrow \infty$.

Let $T_{0}^{\prime}$ be the subset of measure zero of all $t \in G$ where $x_{k}(t)$ does not converge, or does not converge to $x(t)$, or $x(t)$ is not finite. Then $x(t) \in A(t)$ for all $t \in G-$ $\left(T_{0} \cup T_{0}^{\prime}\right)$. For every $s=1,2, \ldots$, let us consider the sequences

$$
\lambda_{s+k}(t), \quad \xi_{s+k}(t), \quad t \in G, \quad k=1,2, \ldots,
$$

which converge weakly to $\lambda(t), \xi(t)$ in $\left(L_{1}(G)\right)^{r+1}$ as $k \rightarrow \infty$. As in the proof of (4.i), by Mazur's theorem there is a set of real numbers $c_{N k}^{(s)} \geqq 0, k=1, \ldots, N$, $N=1,2, \ldots$, with $\sum_{k=1}^{N} c_{N k}^{(s)}=1$, such that if

$$
\lambda_{N}^{(s)}(t)=\sum_{k=1}^{N} c_{N k}^{(s)} \lambda_{s+k}(t), \quad \zeta_{N}^{(s)}(t)=\sum_{k=1}^{N} c_{N k}^{(s)} \xi_{s+k}(t), \quad t \in G, \quad N=1,2, \ldots,
$$

then $\lambda_{N}^{(s)} \rightarrow \lambda$ strongly in $L_{1}(G)$ and $\xi_{N}^{(s)} \rightarrow \xi$ strongly in $\left(L_{1}(G)\right)^{r}$, this being true for every $s=1,2, \ldots$. Then, for every $s$, there is also a subset $T_{s}$ of measure zero of points $t \in G$, and a sequence of integers $N_{h}^{(s)}, h=1,2, \ldots$, with $N_{h}^{(s)} \rightarrow \infty$, such that, for $t \in G-T_{s}$ both $\lambda(t)$ and $\xi(t)$ are finite and $\lambda_{N_{h}}^{(s)}(t) \rightarrow \lambda(t), \xi_{N_{h}}^{(s)}(t) \rightarrow \xi(t)$ as $h \rightarrow \infty$. Let $T$ denote the subset of measure zero in $G$ which is the union of all sets $T_{0}, T_{0}^{\prime}, T_{s}, s=1,2, \ldots$. Note that

$$
\eta_{k}(t) \geqq \lambda_{k}(t), \quad t \in G, \quad \int_{G} \eta_{k}(t) d t=j_{k}, \quad k=1,2, \ldots,
$$


so that, for all $s=1,2, \ldots, N=1,2, \ldots$, we also have

$$
\eta_{N}^{(s)}(t) \geqq \lambda_{N}^{(s)}(t), \quad t \in G, \quad i-\rho_{s} \leqq \int_{G} \eta_{N}^{(s)}(t) d t \leqq i+\rho_{s}
$$

For $N=N_{h}^{(s)}$ and $h \rightarrow \infty$, relations (5.6) and Fatou's lemma imply

$$
\begin{gathered}
\eta^{(s)}(t)=\varliminf_{h \rightarrow \infty} \eta_{N_{h}}^{(s)}(t) \geqq \lambda(t), \quad t \in G, \\
\int_{G} \eta^{(s)}(t) d t \leqq \varliminf_{h \rightarrow \infty} \int_{G} \eta_{N_{h}}^{(s)}(t) d t \leqq i+\rho_{s}, \quad s=1,2, \ldots
\end{gathered}
$$

Thus $\eta^{(s)}(t)$ is finite a.e. in $G$ and of class $L_{1}(G)$.

Let $T_{s}^{\prime}$ denote the set of measure zero of all points $t \in G$ where $\eta^{(s)}(t)$ is not finite. If

then again we have

$$
\eta(t)=\varliminf_{s \rightarrow \infty} \eta^{(s)}(t), \quad t \in G
$$

$$
\eta(t) \geqq \lambda(t), \quad t \in G, \quad \int_{G} \eta(t) d t \leqq i .
$$

Thus, $\eta(t)$ is finite a.e. in $G$ and of class $L_{1}(G)$. Let $T_{0}^{\prime \prime}$ denote the set of measure zero of all points $t \in G$ where $\eta$ is not finite.

Let $T$ denote the set of measure zero in $G$ which is the union of all sets $T_{0}$, $T_{0}^{\prime}, T_{0}^{\prime \prime}, T_{s}, T_{s}^{\prime}, s=1,2, \ldots$ Let $t_{0}$ be any point in $G-T$, and put $x_{0}=x\left(t_{0}\right)$. Then $\left(t_{0}, x_{k}\left(t_{0}\right)\right) \rightarrow\left(t_{0}, x_{0}\right) \in A$, the sets $\tilde{Q}(t, x)$ have property $(Q)$ with respect to $x$ at $\left(t_{0}, x_{0}\right)$, and given $\varepsilon>0$ there is some $s_{0}$ such that $\left|x_{s}\left(t_{0}\right)-x_{0}\right| \leqq \varepsilon$ for $s \geqq s_{0}$. For $s \geqq s_{0}$ we have now

$$
\left(\eta_{s+k}\left(t_{0}\right), \xi_{s+k}(t)\right) \in \tilde{Q}\left(t_{0}, x_{s+k}\left(t_{0}\right)\right), \quad\left|x_{s+k}\left(t_{0}\right)-x_{0}\right| \leqq \varepsilon, \quad k=1,2, \ldots,
$$

and hence

$$
\left(\sum_{k=1}^{N} c_{N k}^{(s)} \eta_{s+k}\left(t_{0}\right), \quad \sum_{k=1}^{N} c_{N k}^{(s)} \xi_{s+k}\left(t_{0}\right)\right) \in c o \underset{x \in N_{\varepsilon, t_{0}}\left(x_{0}\right)}{\bigcup} \tilde{Q}\left(t_{0}, x\right) .
$$

Finally, for $N=N_{h}^{(s)}$ and $h \rightarrow \infty$, the points in the first member of (5.7) form a sequence possessing $\left(\eta\left(t_{0}\right), \xi\left(t_{0}\right)\right)$ as an element of accumulation in $E_{r+1}$ (both $\eta\left(t_{0}\right)$ and $\xi\left(t_{0}\right)$ are finite). Thus

$$
\left(\eta^{(s)}\left(t_{0}\right), \xi\left(t_{0}\right)\right) \in \mathrm{cl} \text { co } \underset{x \in N_{\varepsilon, t_{0}}\left(x_{0}\right)}{\bigcup} \tilde{Q}\left(t_{0}, x\right), \quad s \geqq s_{0} .
$$

Note that $\eta\left(t_{0}\right)=\varliminf_{s \rightarrow \infty} \eta^{(s)}\left(t_{0}\right)$ is finite, so that $\left(\eta\left(t_{0}\right), \xi\left(t_{0}\right)\right)$ is certainly a point of accumulation of the sequence $\left(\eta^{(s)}\left(t_{0}\right), \xi\left(t_{0}\right)\right), s=1,2, \ldots$. Since the second member of (5.8) is closed, we have

$$
\left(\eta\left(t_{0}\right), \xi\left(t_{0}\right)\right) \in \mathrm{cl} \text { co } \underset{x \in N_{\varepsilon, t_{0}}\left(x_{0}\right)}{\bigcup} \tilde{Q}\left(t_{0}, x\right) .
$$

Since $\varepsilon>0$ is arbitrary, by property $(Q)$ we have

$$
\left(\eta\left(t_{0}\right), \xi\left(t_{0}\right)\right) \in \bigcap_{\varepsilon>0} \operatorname{cl} \operatorname{co} \underset{x \in N_{\varepsilon, z_{0}}\left(x_{0}\right)}{\bigcup} \tilde{Q}\left(t_{0}, x\right)=\tilde{Q}\left(t_{0}, x_{0}\right) .
$$


We have shown that $(\eta(t), \xi(t)) \in \tilde{Q}(t, x(t))$ for $t \in G-T$, that is, a.e. in $G$. Statement (5.i) is thereby proved.

Theorem (5.i) has alternate forms of some interest. To formulate them we need a few more notations. For every $(t, x) \in A$ let $Q(t, x)$ denote the projection of $\tilde{Q}(t, x)$ on the $z$-space $E_{r}$. Thus, $\left(z^{0}, z\right) \in \tilde{Q}(t, x)$ implies $z \in Q(t, x)$, and for every $z \in Q(t, x)$ the set $A(t, x, z)=\left[z^{0} \in E_{1} \mid\left(z^{0}, z\right) \in \Phi(t, x)\right]$ is not empty. For every $(t, x) \in A$ let $A^{\prime}(t, x, z)=\operatorname{Inf}\left[z^{0} \mid\left(z^{0}, z\right) \in \tilde{Q}(t, x)\right]$ and

$$
\Lambda^{\prime \prime}(t, x, z)=\operatorname{Sup}\left[z^{0} \mid\left(z^{0}, z\right) \in \hat{Q}(t, x)\right] .
$$

Thus, for every $(t, x) \in A$ and $z \in Q(t, x)$ we have

$$
-\infty \leqq \Lambda^{\prime}(t, x, z) \leqq \Lambda^{\prime \prime}(t, x, z) \leqq \infty,
$$

where $\Lambda^{\prime}$ and $\Lambda^{\prime \prime}$ cannot both be $-\infty$ or both be $+\infty$ at any point.

We shall denote by $\{a, b\}$ any closed interval in $E_{1}$, finite or infinite, that is, any interval of one of the forms $[a, b],[a,+\infty),(-\infty, b],(-\infty,+\infty)$. Note that if $\tilde{Q}(t, x)$ is convex, then also $Q(t, x)$ is convex, and for every $z \in Q(t, x)$ the set $\Lambda(t, x, z)$ is an interval in $E_{1}$. Moreover, if the set $\tilde{Q}(t, x)$ is convex and closed, then for every $z \in Q(t, x)$ we have $\Lambda(t, x, z)=\left\{\Lambda^{\prime}(t, x, z), \Lambda^{\prime \prime}(t, x, z)\right\}$.

We may extend the functions $\Lambda^{\prime}, \Lambda^{\prime \prime}$ to all of $E_{v} \times E_{n} \times E_{r}$ by taking, say, $\Lambda^{\prime}(t, x, z)=\Lambda^{\prime \prime}(t, x, z)=-\infty$ for all $(t, x, z)$ which are not of the form $(t, x) \in A$, $z \in Q(t, x)$. Whenever the sets $\tilde{Q}(t, x)$ are closed and convex, the set valued function $(t, x) \rightarrow \tilde{Q}(t, x)$ is defined by the real valued functions $\Lambda^{\prime}, \Lambda^{\prime \prime}$ (with possible values $-\infty$ and $+\infty$ ). Instead of introducing the usual general concept of measurable set valued functions, we shall simply say here that the set valued function $(t, x) \rightarrow$ $\tilde{Q}(t, x)$ (with values $\tilde{Q}(t, x)$ closed and convex) is $B$-measurable provided the real valued functions $\Lambda^{\prime}, \Lambda^{\prime \prime}$ are $B$-measurable.

We say that the sets $\tilde{Q}(t, x)$ have the upper property provided that for every $(t, x) \in A$ and $\left(\bar{z}^{0}, z\right) \in \tilde{Q}(t, x)$ any other point $\left(z^{0}, z\right)$ with $z^{0} \geqq \bar{z}^{0}$ also belongs to $\tilde{Q}(t, x)$. Thus for closed convex sets $\tilde{Q}(t, x)$ with the upper property, we have $-\infty \leqq \Lambda^{\prime}(t, x, z)<\Lambda^{\prime \prime}(t, x, z)=\infty$ for all $(t, x) \in A$ and $z \in Q(t, x)$.

A real valued function $f(t), t \in Q$, is said to be of class $L^{-}(G)\left[L^{+}(G)\right]$ provided $f$ is measurable on $G$ and its Lebesgue integral $\iint_{G} f(t) d t$ exists and is either finite or $-\infty[+\infty]$. Note that for $f \in L^{-}(G)$ we allow $f$ to attain the value $-\infty$ in any measurable subset of $G$, and the value $+\infty$ in a set of measure zero. An analogous convention holds for $f \in L^{+}(G)$.

We can now formulate the following further theorems which are analogous to statement (5.i):

(5.i)' Let the hypotheses of (5.i) hold, except that the set valued function $(t, x) \rightarrow$ $\tilde{Q}(t, x)$ is B-measurable, and (5.4) is replaced by

$$
\begin{aligned}
& \eta_{k}(t) \geqq \lambda_{k}(t), \quad \lambda_{k}(t) \geqq \lambda_{k+1}(t), \quad \lambda_{k}(t) \rightarrow \lambda(t) \quad \text { pointwise as } k \rightarrow \infty, \\
& \left(\lambda_{k}(t), \xi_{k}(t)\right) \in \tilde{Q}\left(t, x_{k}(t)\right), \quad t \in G(\text { a.e. }), \quad k=1,2, \ldots
\end{aligned}
$$

Then there is a function $\eta(t), t \in G, \eta \in L^{-}(G), \eta(t)$ finite almost everywhere in $G$, such that (5.5) holds. 
Note that in (5.9) the functions $\lambda, \lambda_{k}$ are measurable, but not necessarily $L$-integrable in $G$. Thus (5.9) implies that the functions $\lambda_{k}$ have finite values a.e. in $G$, while $\lambda$ may have the value $-\infty$ even in a set of positive measure, and $\lambda$, $\lambda_{k} \in L^{-}(G)$ (with $\int_{G} \lambda(t) d t \leqq i$, as will be shown in the proof). If $\lambda \in L_{1}(G)$, then also the function $\eta$ in statement $(5 . i)^{\prime}$ is of class $L_{1}(G)$. As will become apparent in the proof of (5.i) the following requirement can replace (5.9): $\lambda, \lambda_{k} \in L^{-}(G)$, $\eta_{k}(t) \geqq \lambda_{k}(t), \quad \lambda_{k}(t) \rightarrow \lambda(t)$ pointwise a.e., $\left(\lambda_{k}(t), \xi_{k}(t)\right) \in \tilde{Q}\left(t, x_{k}(t)\right), \quad t \in G$ (a.e.), $\int_{G} \lambda(t) d t \leqq i$.

(5.i)' Let the hypotheses of $(5.1)^{\prime}$ hold, except that we make the additional requirement :

(5.9)* There is another sequence of functions $\lambda^{\prime \prime}(t), \lambda_{k}^{\prime \prime}(t), t \in G, k=1,2, \ldots$, with

and

$$
\eta_{k}(t) \leqq \lambda_{k}^{\prime \prime}(t), \quad \lambda_{k}^{\prime \prime}(t) \leqq \lambda_{k+1}^{\prime \prime}(t), \quad \lambda_{k}^{\prime \prime}(t) \rightarrow \lambda^{\prime \prime}(t) \text { pointwise as } k \rightarrow \infty,
$$

$$
\left(\lambda_{k}^{\prime \prime}(t), \xi_{k}(t)\right) \in \tilde{Q}\left(t, x_{k}(t)\right), \quad t \in G(\text { a.e. }), \quad k=1,2, \ldots
$$

Then the conclusion of $(5.1)^{\prime}$ holds with $\eta \in L_{1}(G)$.

In particular, we can guarantee that $\eta \in L_{1}(G)$ if we know that the sets $\tilde{Q}(t, x)$ have the upper property.

Proof of (5.i)'. We proceed as in the proof of (5.i), except now we apply MAZUR's theorem to the sequences $\xi_{s+k}(t), t \in G, k=1,2, \ldots$, and we define, as in the proof of (5.i), the real numbers $c_{N k}^{(s)} \geqq 0$, the integers $N_{h}^{(s)}$, and the sets $T_{s}$ of measure zero, $s=1,2, \ldots$.

Note that the functions $\lambda_{k}(t), \lambda(t), t \in G$, are only measurable now. Nevertheless, the relations $\eta_{k}(t) \geqq \lambda_{k}(t), \quad \lambda_{k}(t) \geqq \lambda_{k+1}(t), \lambda_{k}(t) \downarrow \lambda(t)$ imply $\eta_{k}(t) \geqq$ $\lambda_{k}(t) \geqq \lambda(t), t \in G, k=1,2, \ldots$ Since the functions $\eta_{k}$ are of class $L_{1}(G)$, we conclude that the functions $\lambda_{k}, \lambda$ are of class $L^{-}(G)$, where $\lambda$ may have the value $-\infty$ in a set of positive measure. Moreover, for every $k$ we have

$$
-\infty \leqq \int_{G} \lambda(t) d t \leqq \int_{G} \lambda_{k}(t) d t \leqq \int_{G} \eta_{k}(t) d t \leqq i+\rho_{k},
$$

where the first two integrals may have value $-\infty$. For $s=1,2, \ldots$ and $N=1,2, \ldots$, let

so that

$$
\lambda_{N}^{(s)}(t)=\sum_{k=1}^{N} c_{N k}^{(s)} \lambda_{s+k}(t), \quad t \in G,
$$

$$
-\infty \leqq \lambda(t) \leqq \lambda_{N}^{(s)}(t) \leqq \lambda_{s+1}(t) \leqq \eta_{s+1}(t), \quad t \in G .
$$

If we now define

we have again

$$
\begin{aligned}
L^{\prime(s)}(t) & =\varliminf_{h \rightarrow \infty} \lambda_{N_{h}}^{(s)}(t), \quad t \in G, \quad s=1,2, \ldots, \\
L^{\prime}(t) & =\varliminf_{s \rightarrow \infty} L^{(s)}(t), \quad t \in G,
\end{aligned}
$$

$$
-\infty \leqq \lambda(t) \leqq L^{\prime(s)}(t) \leqq \lambda_{s+1}(t) \leqq \eta_{s+1}(t), \quad t \in G
$$


Since $\lambda_{s}(t) \downarrow \lambda(t)$ as $s \rightarrow \infty$, we conclude that $-\infty \leqq \lambda(t)=L^{\prime}(t)<\infty$ for every $t \in G$, and that

$$
-\infty \leqq \int_{G} \lambda(t) d t=\int_{G} L^{\prime}(t) d t \leqq i
$$

Note that for every $(t, x) \in A_{G}-H_{0}$, the sets $Q ⿱ 乛 龰(t, x)$ have property $(Q)$ with respect to $x$; hence they are closed and convex. Therefore for every $z \in Q(t, x)$ we have $\left(z^{0}, z\right) \in \tilde{Q}(t, x)$ for every real number $z^{0},-\infty<z^{0}<\infty$, with $\Lambda^{\prime}(t, x, z) \leqq$ $z^{0} \leqq \Lambda^{\prime \prime}(t, x, z)$. Let

$$
L_{*}(t)=\Lambda^{\prime}(t, x(t), \xi(t)), \quad L^{*}(t)=\Lambda^{\prime \prime}(t, x(t), \xi(t)), \quad t \in G .
$$

Since $\Lambda^{\prime}, \Lambda^{\prime \prime}$ are $B$-measurable, both $L_{*}$ and $L^{*}$ are measurable in $G$. We shall prove below that

$$
-\infty \leqq L_{*}(t) \leqq \lambda(t) \leqq L^{*}(t) \leqq \infty, \quad t \in G-T .
$$

Let $t_{0}$ be any point in $G-T$, and put $x_{0}=x\left(t_{0}\right)$. Since $x_{k}\left(t_{0}\right) \rightarrow x_{0}$ as $k \rightarrow \infty$, we see that, given $\varepsilon>0$, there is some $s_{0}$ such that $s \geqq s_{0}$ implies $\left|x_{k}\left(t_{0}\right)-x_{0}\right|<\varepsilon$. Hence,

$$
\left(\lambda_{s+k}\left(t_{0}\right), \xi_{s+k}\left(t_{0}\right)\right) \in \tilde{Q}\left(t_{0}, x_{s+k}\left(t_{0}\right)\right) \subset S_{\varepsilon}\left(t_{0}\right)
$$

for every $k=1,2, \ldots$, and $s \geqq s_{0}$, where

$$
S_{\varepsilon}\left(t_{0}\right)=\mathrm{clco} \underset{x \in N_{\varepsilon, t_{0}}\left(x_{0}\right)}{\bigcup} \tilde{Q}\left(t_{0}, x\right) .
$$

Then for every $h=1,2, \ldots$ and $s \geqq s_{0}$ we also have

$$
\left(\lambda_{N_{h}}^{(s)}\left(t_{0}\right), \xi_{N_{h}}^{(s)}\left(t_{0}\right)\right) \in S_{\varepsilon}\left(t_{0}\right),
$$

where $\xi_{N_{h}}^{(s)}\left(t_{0}\right) \rightarrow \xi\left(t_{0}\right)$ as $h \rightarrow \infty$. If $\lambda\left(t_{0}\right)$ is finite, so are the numbers $L^{\prime(s)}\left(t_{0}\right)$, $s \geqq s_{0}$, and for every $s \geqq s_{0}$ there is a sequence of integers $\left[h_{\omega}\right]$ such that $h_{\omega} \rightarrow \infty$ and $\lambda_{N_{h}}^{(s)}\left(t_{0}\right) \rightarrow L^{\prime(s)}\left(t_{0}\right)$. Now (5.11) written for $h=h_{\omega}$ yields as $\omega \rightarrow \infty$

$$
\left(L^{(s)}\left(t_{0}\right), \xi\left(t_{0}\right)\right) \in S_{\varepsilon}\left(t_{0}\right), \quad s \geqq s_{0} .
$$

As $\varepsilon \rightarrow 0$ and $s \rightarrow \infty$ we have, by property $(Q)$,

$$
\left(\lambda\left(t_{0}\right), \xi\left(t_{0}\right)\right) \in \bigcap_{\varepsilon>0} S_{\varepsilon}\left(t_{0}\right)=\tilde{Q}\left(t_{0}, x_{0}\right)
$$

and hence $-\infty \leqq L_{*}(t) \leqq \lambda\left(t_{0}\right) \leqq L^{*}\left(t_{0}\right) \leqq \infty$.

If $\lambda\left(t_{0}\right)=-\infty$, choose any fixed point $\left(\bar{z}^{0}, \xi\left(t_{0}\right)\right) \in \tilde{Q}\left(t_{0}, x_{0}\right)$, and another point $z^{0}<\bar{z}^{0}$. Then there is some $s_{1} \geqq s_{0}$ such that for $s \geqq s_{1}$ we have

$$
-\infty=\lambda\left(t_{0}\right) \leqq L^{\prime(s)}\left(t_{0}\right)<z^{0}<\bar{z}^{0}
$$

and again, for every $s \geqq s_{1}$ there is a sequence $\left[h_{\omega}\right]$ of integers, and real numbers $\alpha_{\omega}, 0<\alpha_{\omega}<1$, such that $h_{\omega} \rightarrow \infty, \lambda_{N_{h_{\omega}}}^{(s)}\left(t_{0}\right) \rightarrow L^{\prime(s)}\left(t_{0}\right)$,

$$
\begin{gathered}
L^{(s)}\left(t_{0}\right) \leqq \lambda_{N_{h_{\omega}}}^{(s)}\left(t_{0}\right)<z^{0}<\bar{z}^{0} \leqq L^{*}\left(t_{0}\right), \\
\alpha_{\omega} \lambda_{N_{h_{\omega}}}^{(s)}\left(t_{0}\right)+\left(1-\alpha_{\omega}\right) \bar{z}^{0}=z^{0}, \\
\left(z^{0}, \alpha_{\omega} \xi_{N_{h_{\omega}}}^{(s)}\left(t_{0}\right)+\left(1-\alpha_{\omega}\right) \xi\left(t_{0}\right)\right) \in S_{\varepsilon}\left(t_{0}\right) .
\end{gathered}
$$


As $\omega \rightarrow \infty$, we have

$$
\left.\left(z^{0}, \xi\left(t_{0}\right)\right) \in S_{\varepsilon}\left(t_{0}\right)\right),
$$

and this holds for every $\varepsilon>0$. Thus again, by property $(Q)$, we have

$$
\left(z^{0}, \xi\left(t_{0}\right)\right) \in \bigcap_{\varepsilon>0} S_{\varepsilon}\left(t_{0}\right)=\tilde{Q}\left(t_{0}, x_{0}\right),
$$

and this holds for every $z^{0},-\infty<z^{0}<\bar{z}^{0}$. In other words, $L_{*}\left(t_{0}\right)=\lambda\left(t_{0}\right)=-\infty$. Relation (5.10) has been proved for every $t \in G-T$.

Since $\lambda \in L^{-}(G)$, then for every integer $\mu \geqq 0$ the function

$$
[\lambda(t)]_{-\mu}=\max (\lambda(t),-\mu), \quad t \in G,
$$

is of class $L_{1}(G)$, and we can choose $\mu$ so large that $\int_{G}[\lambda(t)]_{-\mu} d t \leqq i$. Having proved that $-\infty \leqq \lambda(t) \leqq L^{*}(t), t \in G$, we may define $\eta(t), t \in G$, by

$$
\begin{array}{ll}
\eta(t)=\lambda(t) & \text { if }-\mu \leqq \lambda(t) \leqq L^{*}(t) \leqq \infty, \\
\eta(t)=-\mu & \text { if }-\infty \leqq \lambda(t)<-\mu \leqq L^{*}(t) \leqq \infty, \\
\eta(t)=L^{*}(t) & \text { if }-\infty \leqq \lambda(t) \leqq L^{*}(t) \leqq-\mu .
\end{array}
$$

Then $-\infty<\eta(t) \leqq[\lambda(t)]_{-\mu}$; hence $\eta \in L^{-}(G)$ and $\int_{G} \eta(t) d t \leqq i$. On the other hand, we can have $\lambda(t)=L^{*}(t)=\infty$ at most in a set of measure zero, so that $\eta(t)$ is finite almost everywhere in the set of points $t \in G$ where (5.12) occurs. Also, $L^{*}(t)>-\infty$ by definition; hence $\eta(t)$ is always finite in the set of points where (5.14) occurs. Thus $(\eta(t), \xi(t)) \in \tilde{Q}(t, x(t))$ a.e. in $G$.

Proof of (5.i)". The functions $\lambda^{\prime \prime}, \lambda_{k}^{\prime \prime}$ are only measurable, and the relations $\eta_{k}(t) \leqq \lambda_{k}^{\prime \prime}(t) \leqq \lambda_{k+1}^{\prime \prime}, \lambda_{k}^{\prime \prime} \uparrow \lambda^{\prime \prime}$ imply $\eta_{k}(t) \leqq \lambda_{k}^{\prime \prime}(t) \leqq \lambda^{\prime \prime}(t) \leqq \infty, t \in G, \lambda^{\prime \prime}, \lambda_{k}^{\prime \prime} \in L^{+}(G)$; the function $\lambda^{\prime \prime}$ may have the value $+\infty$ in a set of positive measure in $G$. Moreover, we have

$$
i-\rho_{k} \leqq \int_{G} \eta_{k}(t) d t \leqq \int_{G} \lambda_{k}^{\prime \prime}(t) d t \leqq \int_{G} \lambda^{\prime \prime}(t) d t \leqq \infty .
$$

As in the proof of $(5 . i)^{\prime}$ we put

so that

$$
\begin{aligned}
L^{\prime \prime(s)}(t) & =\varlimsup_{h \rightarrow \infty} \lambda_{N_{h}}^{\prime \prime(s)}(t), \quad t \in G, \quad s=1,2, \ldots, \\
L^{\prime \prime}(t) & =\varlimsup_{s \rightarrow \infty} L^{\prime \prime(s)}(t), \quad t \in G,
\end{aligned}
$$

$$
\eta_{s+1}(t) \leqq \lambda_{s+1}^{\prime \prime}(t) \leqq L^{\prime \prime(s)}(t) \leqq \lambda^{\prime \prime}(t) \leqq \infty, \quad t \in G .
$$

Since $\lambda_{s}^{\prime \prime}(t) \uparrow \lambda^{\prime \prime}(t), t \in G$, as $s \rightarrow \infty$, we conclude that

$$
\begin{gathered}
-\infty \leqq L^{\prime \prime}(t)=\lambda^{\prime \prime}(t) \leqq \infty, \quad t \in G, \\
i \leqq \int_{G} L^{\prime \prime}(t) d t=\int_{G} \lambda^{\prime \prime}(t) d t \leqq \infty
\end{gathered}
$$

Finally, we can prove as in (5.i)' that

$$
-\infty \leqq L_{*}(t) \leqq \lambda(t) \leqq \lambda^{\prime \prime}(t) \leqq L^{*}(t) \leqq \infty, \quad t \in G-T .
$$


Now we have $\lambda \in L^{-}(G), \lambda^{\prime \prime} \in L^{+}(G)$; we define $\mu$ as in the proof of (5.i)', and we define $\eta(t), t \in G$, by

$$
\begin{array}{ll}
\eta(t)=\lambda(t) & \text { if }-\mu \leqq \lambda(t) \leqq \lambda^{\prime \prime}(t) \leqq \infty, \\
\eta(t)=-\mu & \text { if }-\infty \leqq \lambda(t) \leqq-\mu \leqq \lambda^{\prime \prime}(t) \leqq \infty, \\
\eta(t)=\lambda^{\prime \prime}(t) & \text { if }-\infty \leqq \lambda(t) \leqq \lambda^{\prime \prime}(t) \leqq-\mu .
\end{array}
$$

If $S_{1}, S_{2}, S_{3}$ denote the sets of points $t \in G$ where the cases (5.15), (5.16), (5.17) occur, then $\lambda(t) \geqq-\mu$ on $S_{1}$ and the fact that $\lambda \in L^{-}(G)$ implies $\lambda \in L_{1}\left(S_{1}\right)$. Also $\eta$ is a constant on $S_{2}$. Finally $\lambda^{\prime \prime} \in L^{+}(G), \lambda^{\prime \prime} \leqq-\mu$ on $S_{3}$ so that $\lambda^{\prime \prime} \in L_{1}\left(S_{3}\right)$; hence $\eta \in L_{1}\left(S_{3}\right)$. Thus $\eta \in L_{1}(G)$. The relation $\eta(t) \leqq[\lambda(t)]_{-\mu}$ implies $\int_{G} \eta(t) d t \leqq i$
as in the proof of $(5.1)^{\prime}$, but now we know that $\eta \in L_{1}(G)$.

Note that if the sets $\tilde{Q}(t, x)$ have the upper property, then we can take $\lambda_{k}^{\prime \prime}(t)=$ $\operatorname{Max}\left[\eta_{1}(t), \ldots, \eta_{k}(t)\right], t \in G, k=1,2, \ldots$, and the sequence $\lambda_{k}^{\prime \prime}$ satisfies all requirements of (5.i)".

Remark 6. In statement (5.i), the requirement (5.4) cannot be disregarded, even if we replace (5.2) by the stronger condition $\int_{G}\left|\eta_{k}(t)\right| d t \leqq M_{0}$. This is shown by the following example. Take $v=n=r=1,0 \leqq t \leqq 1,0 \leqq x \leqq 1, \quad \tilde{Q}(t, x)=$ $\left[\left(z^{0}, z\right) \mid z^{0} \geqq 0, z=0\right]$ if $0 \leqq t<1,0 \leqq x \leqq 1, t+x<1 ; \bar{Q}(t, x)=\left[\left(z^{0}, z\right) \mid z^{0} \geqq-x^{-1}\right.$, $z=0]$ if $0 \leqq t<1,0<x \leqq 1, t+x \geqq 1 ; \tilde{Q}(1, x)=E_{1} \times\{0\}$. Then all sets $\tilde{Q}(t, x)$ are closed half lines, or lines, and have property $(Q)$ with respect to $x$ everywhere. Let us take $\xi_{k}(t)=\xi(t)=0, x_{k}(t)=k^{-1}, x(t)=0,0 \leqq t \leqq 1, \eta_{k}(t)=0$ for $0 \leqq t<1-k^{-1}$ and for $t=1, \eta_{k}(t)=-k$ for $1-k^{-1} \leqq t<1$. Then $\int_{0}^{1} \eta_{k}(t) d t=-1$ and $\int_{0}^{1}\left|\eta_{k}(t)\right| d t=1$, $k=1,2, \ldots$. For $x(t)=0,0 \leqq t \leqq 1$, we must have $\eta(t) \geqq 0$ for all $0 \leqq t<1$. Hence $\int_{0}^{1} \eta(t) d t \geqq 0$, and relations (5.5) cannot be satisfied.

This example also shows that condition (5.9) cannot be disregarded in (5.i)'. Here the sets $\tilde{Q}(t, x)$ have the upper property, and condition (5.9)* is certainly satisfied.

(5.ii) Let the hypotheses of (5.i) hold, except that the subsets $\tilde{Q}(t, x)$ of $E_{r+1}$ satisfy only property $(K)$ with respect to $x$ in $A_{G}-H_{0}$, and $\xi_{k}(t) \rightarrow \xi(t)$ pointwise a.e. in $G$ as $k \rightarrow \infty$. Then the conclusion of (5.i) also holds.

Again, as for statement (5.i), functions $\lambda, \lambda_{k}$ satisfying (5.4) can be found immediately (with the consequent inequality $i>-\infty$ ) under the conditions $(\alpha)$, $(\beta)$ with $\gamma=0,(\gamma)$ with $\gamma=0$, and $(\delta)$ of Remark 5 above.

Conditions $(\beta)$ and $(\gamma)$ with $\gamma>0$ do not apply, however. To see this we may consider the counterexample of Remark 6 with the following modification. Define $Q \tilde{Q}(t, x)$ as in Remark 6 for $0 \leqq t<1,0 \leqq x \leqq 1, t+x<1$, and for $t=1$, $0 \leqq x \leqq 1$, but take instead $\tilde{Q}(t, x)=\left[\left(z^{0}, z\right) \mid z^{0} \geqq-x^{-1}, z=-x^{-1}\right]$ for $0 \leqq t<1$, $0<x<1, t+x \geqq 1$. Correspondingly, choose $\eta_{k}(t)$ as in Remark 6, and put $\xi_{k}(t)=\eta_{k}(t), \xi(t)=0,0 \leqq t \leqq 1$. Now $\xi_{k}(t) \rightarrow \xi(t)$ pointwise in $[0,1]$ as $k \rightarrow \infty$ and condition $(\beta)$ holds for $\psi(t)=0, \gamma=1$, yet the conclusions of (5.i, ii) do not hold. 
Remark 7. Theorem (5.iii) below concerns the case where the sequences $\eta_{k}(t)$, $\xi_{k}(t), x_{k}(t), \quad t \in G, k=1,2, \ldots$, satisfying $\left(\eta_{k}(t), \xi_{k}(t)\right) \in \tilde{Q}\left(t, x_{k}(t)\right), t \in G$ (a.e.), with $\xi_{k} \rightarrow \xi$ weakly in $\left(L_{1}(G)\right)$, $\varliminf_{G} \eta_{k}(t) d t=i, x_{k}(t) \rightarrow x(t)$ a.e. in $G$ as $k \rightarrow \infty$, can be replaced by a modified sequence $\bar{\eta}_{k}(t), \bar{\xi}_{k}(t), x(t), t \in G, k=1,2, \ldots$, satisfying $\left(\bar{\eta}_{k}(t), \bar{\xi}_{k}(t)\right) \in \tilde{Q}(t, x(t)), t \in G$ (a.e.), where $\bar{\xi}_{k}$ still converges to $\xi$ weakly in $\left(L_{1}(G)\right)^{r}$ and $\varliminf_{G} \int_{G} \bar{\eta}_{k}(t) d t=i$. In other words, we need to know that $\delta_{k}(t)=\xi_{k}(t)-\bar{\xi}_{k}(t) \rightarrow 0$ weakly in $\left(L_{1}(G)\right)^{r}$ and $\delta_{k}^{0}(t)=\eta_{k}(t)-\bar{\eta}_{k}(t) \rightarrow 0$ weakly in $L_{1}(G)$ as $k \rightarrow \infty$. This situation actually occurs in a large class of problems (see Remark 12 below), and in this situation the sets $\tilde{Q}(t, x)$ need be assumed only convex and closed (properties $(Q)$ or $(K)$ are not required).

(5.iii) Let the hypotheses of (5.i) hold, except that the subsets $\hat{Q}(t, x)$ of $E_{r+1}$ are only assumed to be closed and convex for every $(t, x) \in A_{G}-H_{0}$, where $\xi_{k} \rightarrow \xi$ weakly in $\left(L_{1}(G)\right)^{r}$ as $k \rightarrow \infty$, and $x_{k}(t)=x(t)$ for all $t$ and $k$. Then the conclusion of (5.i) holds.

For this statement (5.ii) too there are functions $\lambda, \lambda_{k}$ satisfying (5.4), with consequent inequality $i>-\infty$, under the same conditions $(\alpha)$, or $(\beta)$ with $\gamma \geqq 0$, or $(\gamma)$ with $\gamma \geqq 0$, or $(\delta)$ of Remark 5 above (exactly as for (5.i)).

\section{Closure Theorems for Mayer Problems}

We shall use essentially the notation of $\S 2$. Points in $E_{v}, E_{n}, E_{m}, E_{\mathrm{r}}$ spaces will be denoted by $t=\left(t^{1}, \ldots, t^{v}\right), x=\left(x^{1}, \ldots, x^{n}\right), u=\left(u^{1}, \ldots, u^{m}\right)$, and $z=\left(z^{1}, \ldots, z^{n}\right)$, or $\xi=\left(\xi^{1}, \ldots, \xi\right)$, respectively. Also as before $A$ is a subset of $E_{v} \times E_{n}, A_{0}$ is the projection of $A$ on $E_{v}$, and $A(t)=\left[x \in E_{n} \mid(t, x) \in A\right]$. A subset $U(t, x)$ of $E_{m}$ is assigned for every $(t, x) \in A$, and we put $M=[(t, x, u) \mid(t, x) \in A, u \in U(t, x)] \subset E_{v} \times$ $E_{n} \times E_{m}$. Here $f(t, x, u)=\left(f_{1}, \ldots, f_{r}\right)$ is a given function on $M$, and, for every $(t, x) \in A, Q(t, x)$ will denote the subset of $E_{r}$ defined by

$$
Q(t, x)=f(t, x, U(t, x))=\left[z \in E_{r} \mid z=f(t, x, u), u \in U(t, x)\right] .
$$

We shall denote by $G$ and $T_{0}$ given subsets of $A_{0}$ in $E_{v}$; then $A_{G}$ and $H_{0}$ are the sets $A_{G}=A \cap\left(G \times E_{n}\right)$ and $H_{0}=A \cap\left(T_{0} \times E_{n}\right)$. Finally we shall denote by $x(t)=$ $\left(x^{1}, \ldots, x^{n}\right), u(t)=\left(u^{1}, \ldots, u^{m}\right), \xi(t)=\left(\xi^{1}, \ldots, \xi\right), t \in G$, given functions on $G$.

Measurable functions $x(t), t \in G$, with values $x(t) \in A(t)$ a.e. in $G$, are said to be state functions; measurable functions $u(t), t \in G$, with values $u(t) \in U(t, x(t))$ a.e. in $G$, are said to be controls relative to the state function $x(t), t \in G$.

In [1 $\mathrm{ab}]$ we have already proved closure theorems for Mayer problems for $v=1$ by direct application of statement (3.i) (or variants of this given in Remark 2) and the use of the MCSHANE-WARFIELD implicit function theorem [10]. Analogously, we have proved [1 ghi] similar closure theorems for $v>1$. We shall not, however, discuss them here.

In the following we shall need only a very mild closure and continuity requirement on $A, M$, and $f$, which we refer to as property $(C)$ (Carathéodory type condition). We shall say that condition $(C)$ is satisfied, provided that given an $\varepsilon>0$ there is a compact subset $K$ of $G$ such that $|G-K|<\varepsilon$, the sets $A_{R}=$ $[(t, x) \in A \mid t \in K]$ and $M_{K}=[(t, x, u) \in M \mid t \in K]$ are closed, and $f(t, x, u)$ is contin- 
uous on $M_{K}$. This requirement will suffice for the application of the MCSHANEWARFIELD theorem. On the other hand, property $(C)$ certainly implies that the sets $A(t)$ are closed for almost all $t \in G$. For the sake of brevity, we shall say that $f$ satisfies property $(C)$ when the above conditions are satisfied.

Statements (4.i), (4.ii), (4.iii) and the McShane-Warfield theorem yield the following statements (6.i-iii).

(6.i) If $G$ is a measurable set with finite measure and $T_{0}$ has measure zero, if $f(t, x, u)$ satisfies the continuity property $(C)$ on $M$, if the corresponding sets $Q(t, x)=f(t, x, U(t, x))$ defined above satisfy property $(Q)$ with respect to $x$ in $A_{G}-H_{0}$, if $\xi(t), x(t), \xi_{k}(t), x_{k}(t), u_{k}(t), t \in G, k=1,2, \ldots$, are measurable functions, $\xi, \xi_{k} \in\left(L_{1}(G)\right)^{r}$, if

$$
\begin{gathered}
x_{k}(t) \in A(t), \quad u_{k}(t) \in U\left(t, x_{k}(t)\right), \\
\xi_{k}(t)=f\left(t, x_{k}(t), u_{k}(t)\right), \quad t \in G(\text { a.e. }), \quad k=1,2, \ldots,
\end{gathered}
$$

and if $x_{k}(t) \rightarrow x(t)$ in measure in $G$ and $\xi_{k} \rightarrow \xi$ weakly in $\left(L_{1}(G)\right)^{r}$ as $k \rightarrow \infty$, then there is some measurable function $u(t), t \in G$, such that

$$
x(t) \in A(t), \quad u(t) \in U(t, x(t)), \quad \xi(t)=f(t, x(t), u(t)), \quad t \in G \text { (a.e.). }
$$

Note that for (6.i) to hold we do not need the full force of property $(Q)$ with respect to $x$. All we need is the existence of a subsequence $k_{s}$ such that $x_{k_{g}}(t) \rightarrow x(t)$ pointwise a.e. in $G$, and such that for almost all $t \in G$ we have

$$
Q(t, x(t)) \supset \bigcap_{h=1}^{\infty} \operatorname{clco}\left\{\bigcup_{s=h}^{\infty} f\left(t, x_{k_{s}}(t), u_{k_{s}}(t)\right)\right\} .
$$

The same remark holds for theorem (6.ii) below.

Remark 8. Statement (6.i) has the usual modifications in the situation of Remark 1 with $v=1, r=n, \operatorname{cl} G=[a, b]=a$ fixed interval in $E_{1}, x, x_{k} A C, \xi(t)=x^{\prime}(t)$, $\xi_{k}(t)=x_{k}^{\prime}(t), t \in[a, b]$ (a.e.), and $\left(t, x_{k}(t)\right) \in A, u_{k}(t) \in U\left(t, x_{k}(t)\right), x_{k}^{\prime}(t)=f\left(t, x_{k}(t)\right.$, $\left.u_{k}(t)\right), t \in[a, b]$ (a.e.), $k=1,2, \ldots$. Again, if the sets $Q(t, x)$ have property $(Q)$ with respect to $x$ only, if $x_{k}^{\prime} \rightarrow x^{\prime}$ weakly in $L_{1}([a, b])$, and if $x_{k}(\bar{t}) \rightarrow x(\bar{t})$ at least at one point $\bar{t}$, then the uniform convergence $x_{k} \rightarrow x$ in $[a, b]$ follows, and there is a measurable function $u(t), t \in[a, b]$, such that $(t, x(t)) \in A, u(t) \in U(t, x(t))$, $x^{\prime}(t)=f(t, x(t), u(t)), t \in[a, b]$ (a.e.).

(6.ii) Let the hypotheses of (6.i) hold, except that the sets $Q(t, x)$ satisfy only property $(K)$ with respect to $x$ in $A_{G}-H_{0}$, where $\xi, \xi_{k}$ are only measurable, and where $\xi_{k}(t) \rightarrow \xi(t), x_{k}(t) \rightarrow x(t)$ pointwise a.e. in $G$ as $k \rightarrow \infty$. Then the conclusion of (6.i) holds.

If we assume that the sets $U(t)$ depend on $t$ only, if $\xi(t), \xi_{k}(t), x(t), x_{k}(t)$, $u_{k}(t), t \in G, k=1,2, \ldots$, are measurable functions, and if, as usual, $x_{k}(t) \in A(t)$, $u_{k}(t) \in U(t), \xi_{k}(t)=f\left(t, x_{k}(t), u_{k}(t)\right), t \in G$ (a.e.), $k=1,2, \ldots$, we may consider the auxiliary functions $\bar{\xi}_{k}(t)=f\left(t, x(t), u_{k}(t)\right), t \in G$, and the differences

$$
\delta_{k}(t)=f\left(t, x_{k}(t), u_{k}(t)\right)-f\left(t, x(t), u_{k}(t)\right), \quad t \in G, \quad k=1,2, \ldots,
$$

which exist because $U(t)$ depends on $t$ only. In view of Remark 3 we should try to prove that $\delta_{k} \rightarrow 0$ weakly in $\left(L_{1}(G)\right)^{r}$. Actually, there are natural hypotheses 
which guarantee that $\delta_{k} \rightarrow 0$ strongly in $\left(L_{1}(G)\right)^{r}$. Also the following statement was proved in [3]:

(*) Solely under the Carathéodory type continuity condition $(C)$, if $x(t)$, $x_{k}(t), u_{k}(t), t \in G$, are measurable functions, $u_{k} \in\left(L_{1}(G)^{m}\right),\left\|u_{k}\right\|_{1} \leqq M_{0}=$ constant, $x(t), x_{k}(t) \in A(t), \quad u_{k}(t) \in U(t), \quad t \in G$ (a.e.), $k=1,2, \ldots$, and if $x_{k}(t) \rightarrow x(t)$ in measure in $G$ as $k \rightarrow \infty$, then $\delta_{k} \rightarrow 0$ strongly in $\left(L_{1}(G)\right)^{r}$ if and only if the same functions $\delta_{k}(t), t \in G, k=1,2, \ldots$, are equiabsolutely integrable in $G$.

In Remark 9 below we list a few conditions under which $\delta_{k} \rightarrow 0$ strongly in $\left(L_{1}(G)\right)^{r}$. Further conditions, details and proofs are given in [3].

The following simple closure theorem, in which no condition $(Q)$ or $(K)$ is required, is now a corollary of (4.iii). Instead of the orientor field relations $\xi_{k}(t) \in Q\left(t, x_{k}(t)\right), t \in G$ (a.e.), we simply consider the orientor field relations $\bar{\xi}_{k}(t) \in Q(t, x(t)), t \in G$ (a.e.), $k=1,2, \ldots$, and apply (4.iii).

(6.iii) Let the hypotheses of (6.i) hold, except that the sets $U(t)$ depend on $t$ only, the sets $Q(t, x)$ are assumed to be only convex and closed for every $(t, x) \in$ $A_{G}-H_{0}$, where $\xi, \xi_{k}, \delta_{k} \in\left(L_{1}(G)\right)^{r}, \xi_{k} \rightarrow \xi, \delta_{k} \rightarrow 0$ weakly in $\left(L_{1}(G)\right)^{r}$, and $x_{k}(t) \rightarrow x(t)$ pointwise a.e. in $G$ as $k \rightarrow \infty$. Then the conclusion of (6.i) holds.

Remark 9. A great many particular conditions guarantee that $\delta_{k} \rightarrow 0$ strongly in $\left(L_{1}(G)\right)^{r}$. We mention here, for example, Lipschitz-type hypotheses such as $\left(F_{p}\right),\left(F_{\infty}\right)$ below, and growth-type hypotheses such as $\left(G_{p q}\right),\left(G_{\infty q}\right)$ below. The latter type has been proposed by F. E. BrowDer (Arch. Rational Mech. Anal. 20, 1965, 251-258) and by E. H. RotHe (Arch. Rational Mech. Anal. 21, 1966, 151-162) for free problems of the calculus of variations. We list briefly some of these hypotheses here, referring to [3] for further conditions and details. In any case, we assume that $f$ satisfies the continuity condition $(C)$.

$\left(F_{p}\right)$ For $1 \leqq p<\infty$, we have $x_{k}, x \in\left(L_{p}(G)\right)^{n},\left\|x_{k}-x\right\|_{p} \rightarrow 0$, and

$$
\left|f\left(t, x_{k}(t)\right)-f\left(t, x(t), u_{k}(t)\right)\right| \leqq F\left(t, u_{k}(t)\right) h\left(\left|x_{k}(t)-x(t)\right|\right), \quad t \in G, \quad k=1,2, \ldots,
$$

where $h(\xi) \geqq 0,0 \leqq \xi<+\infty$, is a given monotone nondecreasing function with $h(0+)=0, h(\xi) \leqq c \xi$ for all $\xi>\xi_{0} \geqq 0$, and $F\left(t, u_{k}(t)\right) \in L_{p^{\prime}}(G), 1 / p^{\prime}+1 / p=1$, $\left\|F\left(t, u_{k}(t)\right)\right\|_{p^{\prime}} \leqq C$, where $\xi_{0}, c, C$ are constants and $F(t, u)$ is a given non-negative function defined on $G \times E_{m}$.

$\left(F_{\infty}\right)$ We have $x_{k}, x \in L_{\infty}(G),\left\|x_{k}-x\right\|_{\infty} \rightarrow 0$, and

$$
\left|f\left(t, x_{k}(t), u_{k}(t)\right)-f\left(t, x(t), u_{k}(t)\right)\right| \leqq F\left(t, u_{k}(t)\right) h\left(\left|x_{k}(t)-x(t)\right|\right), \quad t \in G,
$$

where $h$ is as in $\left(F_{p}\right)$, and $F\left(t, u_{k}(t)\right) \in L_{1}(G), \int_{G} F\left(t, u_{k}(t)\right) d t \leqq C$.

$\left(G_{p q}\right)$ For $1 \leqq p, q<\infty, x_{k}, x \in\left(L_{p}(G)\right)^{n}, u_{k}, u \in\left(L_{p}(G)\right)^{m},\|x\|_{p},\left\|x_{k}\right\|_{p} \leqq L_{0},\|u\|_{q}$, $\left\|u_{k}\right\|_{q} \leqq L, L_{0}, L$ given constants, we have $x_{k}(t) \rightarrow x(t)$ pointwise a.e. in $G$ as $k \rightarrow \infty$, and there are constants $c, c^{\prime}, \alpha, \beta, 0<\alpha \leqq p, 0<\beta \leqq q$, and a function $\psi(t) \geqq 0, t \in G, \psi \in L_{1}(G)$, such that for all $(t, x, u),(t, y, u) \in M$ we have

$$
|f(t, x, u)-f(t, y, u)| \leqq \psi(t)+c\left(|x|^{p-\alpha}+|y|^{p-\alpha}\right)+c^{\prime}|u|^{q-\beta} .
$$

$\left(G_{\infty q}\right)$ For $1 \leqq q<\infty, x, x_{k} \in\left(L_{\infty}(G)\right)^{n}, u, u_{k} \in\left(L_{q}(G)\right)^{m},\|x\|_{\infty},\left\|x_{k}\right\|_{\infty} \leqq L_{0},\|u\|_{q}$, $\left\|u_{k}\right\|_{q} \leqq L, L, L_{0}$ given constants, we have $x_{k}(t) \rightarrow x(t)$ pointwise a.e. in $G$ as 
$k \rightarrow \infty$, and there are constants $c^{\prime}, \beta, 0<\beta \leqq q$, a function $\psi(t) \geqq 0, t \in G, \psi \in L_{1}(G)$, and a monotone nondecreasing function $\sigma(\xi) \geqq 0,0 \leqq \xi<+\infty$, such that for all $(t, x, u),(t, y, u) \in M$ we have

$$
|f(t, x, u)-f(t, y, u)| \leqq \psi(t)(\sigma(|x|)+\sigma(|y|))+c^{\prime}|u|^{q-\beta} .
$$

Note that in $\left(G_{p q}\right),\left(G_{\infty q}\right)$ we do not assume that $\sigma(0+)=0$. These conditions are only growth conditions, the continuity of $f$ (property $(C)$ having the main role in the proof that $\delta_{k} \rightarrow 0$ ). Note that, for $f$ linear in $x$, that is, of the form $f(t, x, u)=B(t, u) x+C(t, u)$, where $B=\left[b_{i j}(t, x)\right], C=\left[c_{i}(t, x)\right]$ are matrices of the types $r \times n, r \times 1$, with $\left|b_{i j}(t, u)\right| \leqq \Phi(t)$ for $\Phi(t) \geqq 0, t \in G, \Phi \in L_{p^{\prime}}(G)$, condition $\left(F_{p}\right)$ is certainly satisfied.

Remark 10. In applications (see, e.g., [1 ij], [2]) we may have to consider a topological space $S$ of elements $y$, operators $L: S \rightarrow\left(L_{1}(G)\right)^{r}, M: S \rightarrow\left(L_{1}(G)\right)^{n}$, and a class $T$ of measurable controls $u(t), t \in G$, such that if $x(t)=(M y)(t)$, $\xi(t)=(L y)(t), \quad t \in G$, then $x(t) \in A(t), u(t) \in U(t, x(t)), \xi(t)=f(t, x(t), u(t))$, $t \in G$ (a.e.), that is, we may have to satisfy the constraints $(M y)(t) \in A(t), u(t) \in$ $U(t,(M y)(t))$, and the system of equations $(L y)(t)=f(t,(M y)(t), u(t)), t \in G($ a.e. $)$. We then take into consideration a functional $I[y, u]$, or $I: \Omega \rightarrow E_{1}$, defined in a suitable subset $\Omega$ of $S \times T$. If the functional $I$ can be expressed in terms of $x$ only, that is, if $I$ is of the form $I[x]$, we may say that we have an abstract Mayer problem. As shown in [1 ijk] suitable requirements on $L$ and $M$ are as follows: If $y_{k} \rightarrow y$ in $S$ (that is, in the topology of $S$ ), then $L y_{k} \rightarrow L y$ weakly in $\left(L_{1}(G)\right)^{r}$, and $M y_{k} \rightarrow$ $M y$ strongly in $\left(L_{1}(G)\right)^{n}$, that is, $\xi_{k} \rightarrow \xi$ weakly, and $x_{k} \rightarrow x$ strongly. Then, a corresponding requirement on the Mayer functional $I[x]$ is that $I\left[x_{k}\right] \rightarrow I[x]$ as $k \rightarrow \infty$. Closure theorems of the present $\S 6$ apply to abstract Mayer problems of this type. We refer to [ $1 \mathrm{ijk}$ ] for details and more general formulations.

\section{Lower Closure Theorems for Lagrange Problems}

We shall use essentially the notations of $\S 2$. Points in $E_{v}, E_{n}, E_{m}, E_{r+1}$ spaces will be denoted by $t=\left(t^{1}, \ldots, t^{v}\right), x=\left(x^{1}, \ldots, x^{m}\right), u=\left(u^{1}, \ldots, u^{m}\right)$, and $\left(z^{0}, z\right)=$ $\left(z^{0}, z^{1}, \ldots, z^{r}\right)$, or $(\eta, \xi)=\left(\eta, \xi^{1}, \ldots, \xi^{r}\right)$, respectively. Also, $A$ is a subset of $E_{y} \times E_{n}$, $A_{0}$ the projection of $A$ on $E_{v}$, and $A(t)=\left[x \in E_{n} \mid(t, x) \in A\right]$. For every $(t, x) \in A$ a subset $U(t, x)$ of $E_{m}$ is assigned, and $M$ denotes the set $[(t, x, u) \mid(t, x) \in A$, $u \in U(t, x)] \subset E_{v} \times E_{n} \times E_{m}$. Here $f_{0}(t, x, u), f(t, x, u)=\left(f_{1}, \ldots, f_{r}\right)$ are given functions on $M$, and, for every $(t, x) \in A, \tilde{Q}(t, x)$ denotes the subset of $E_{r+1}$ defined by

$$
\tilde{Q}(t, x)=\left[\left(z^{0}, z\right) \mid z^{0} \geqq f_{0}(t, x, u), z=f(t, x, u), u \in U(t, x)\right] .
$$

We shall denote by $G$ and $T_{0}$ given subsets of $A_{0}$ in $E$; then $A_{G}$ and $H_{0}$ are the sets $A_{G}=A \cap\left(G \times E_{n}\right), H_{0}=A \cap\left(T_{0} \times E_{n}\right)$. Finally, we shall denote by $x(t)=$ $\left(x^{1}, \ldots, x^{n}\right), u(t)=\left(u^{1}, \ldots, u^{m}\right), \xi(t)=\left(\xi^{1}, \ldots, \xi^{m}\right), \eta(t), t \in G$, given functions on $G$.

(7.i) If $G$ is a measurable set with finite measure, and $T_{0}$ has measure zero, if $f_{0}(t, x, u), f(t, x, u)=\left(f_{1}, \ldots, f_{r}\right)$ satisfy the continuity property $(C)$ on $M$, if the corresponding subsets $\tilde{Q}(t, x)$ of $E_{r+1}$ defined above satisfy property $(Q)$ with respect to $x$ in $A_{G}-H_{0}$, if $\xi(t), x(t), \xi_{k}(t), \eta_{k}(t), x_{k}(t), u_{k}(t), \lambda(t), \lambda_{k}(t), t \in G$, 
$k=1,2, \ldots$, are measurable functions, $\xi, \xi_{k} \in\left(L_{1}(G)\right)^{r}, \eta_{k} \in L_{1}(G)$, if

$$
\begin{gathered}
x_{k}(t) \in A(t), \quad u_{k}(t) \in U\left(t, x_{k}(t)\right), \\
\xi_{k}(t)=f\left(t, x_{k}(t), u_{k}(t)\right), \quad \eta_{k}(t)=f_{0}\left(t, x_{k}(t), u_{k}(t)\right) \\
-\infty<i=\varliminf_{k \rightarrow \infty} \int_{G} \eta_{k}(t) d t<\infty,
\end{gathered}
$$

$\xi_{k} \rightarrow \xi$ weakly in $\left(L_{1}(G)\right)^{r}, \quad x_{k}(t) \rightarrow x(t)$ in measure in $G$ as $k \rightarrow \infty$,

$$
\eta_{k}(t) \geqq \lambda_{k}(t), \quad \lambda, \lambda_{k} \in L_{1}(G), \quad \lambda_{k} \rightarrow \lambda \text { weakly in } L_{1}(G),
$$

then there is some measurable function $u(t), t \in G$, such that, if $\eta(t)=f_{0}(t, x(t), u(t))$, $t \in G$, then $\eta \in L^{-}(G)$, and

$$
\begin{gathered}
x(t) \in A(t), \quad u(t) \in U(t, x(t)), \quad \xi(t)=f(t, x(t), u(t)), \quad t \in G \text { (a.e.), } \\
\int_{G} \eta(t) d t \leqq i=\lim _{k \rightarrow \infty} \int_{G} \eta_{k}(t) d t .
\end{gathered}
$$

Proof. In view of (5.i) there is a function $\bar{\eta}(t), t \in G, \bar{\eta} \in L_{1}(G)$, such that

$$
x(t) \in A(t), \quad(\bar{\eta}(t), \xi(t)) \in \tilde{Q}(t, x(t)), \quad t \in G, \text { (a.e.), } \quad \int_{\tilde{G}} \bar{\eta}(t) d t \leqq i .
$$

By the McShane-Warfield theorem there is a measurable function $u(t), t \in G$, such that

$$
\begin{array}{cl}
x(t) \in A(t), \quad u(t) \in U(t, x(t)), & \xi(t)=f(t, x(t), u(t)), \\
f_{0}(t, x(t), u(t)) \leqq \bar{\eta}(t), & t \in G(\text { a.e. }) .
\end{array}
$$

Finally, by taking

we have

$$
\eta(t)=f_{0}(t, x(t), u(t)), \quad t \in G
$$

$$
\eta(t) \leqq \bar{\eta}(t), \quad t \in G, \quad \int_{G} \eta(t) d t \leqq \int_{G} \bar{\eta}(t) d t \leqq i,
$$

and $\eta$ is certainly measurable and of class $L^{-}(G)$. In any case, relations (7.5) hold, and statement (7.i) is proved.

Note that, for (7.i) to hold, we do not need the full force of property $(Q)$ with respect to $x$. All we need is that there is a subsequence $k_{s}$ such that $x_{k_{s}}(t) \rightarrow x(t)$ pointwise a.e. in $G$, and such that, for almost all $t \in G$, we have

$$
\tilde{Q}(t, x(t)) \supset \bigcap_{h=1}^{\infty} \operatorname{cl} \operatorname{co}\left\{\bigcup_{s=h}^{\infty} \tilde{f}\left(t, x_{k_{s}}(t), u_{k_{s}}(t)\right)\right\} \text {. }
$$

where $\tilde{f}=\left(f_{0}, f\right)=\left(f_{0}, f_{1}, \ldots, f_{r}\right)$. The same remark holds for theorem (7.ii) below

Remark 11. Requirement (7.4) and the $L$-integrability of $\eta$ in statement (7.i) can be guaranteed under a variety of conditions, for instance, any of the following.

$\left(\alpha_{0}\right)$ There is a real valued function $\psi(t) \geqq 0, t \in G, \psi \in L_{1}(G)$, such that $f_{0}(t, x, u) \geqq-\psi(t)$ for all $(t, x, u) \in M$.

Indeed, as in $\S 5$ under $(\alpha)$ of Remark 5 , we have $\eta_{k}(t) \geqq \lambda_{k}(t)=-\psi(t), t \in G$, $k=1,2, \ldots$. Then in the proof of (7.i) we have $-\psi(t) \leqq \eta(t)=f_{0}(t, x(t), u(t)) \leqq \bar{\eta}(t)$, $t \in G$, where both $\psi$ and $\bar{\eta}$ are $L$-integrable; hence $\eta \in L_{1}(G)$. 
$\left(\beta_{0}\right)$ There exists a real valued function $\psi(t) \geqq 0, t \in G, \psi \in L_{1}(G)$, and a constant $\gamma \geqq 0$ such that $f_{0}(t, x, u) \geqq-\psi(t)-\gamma|f(t, x, u)|$ for all $(t, x, u) \in M$.

Indeed, as in $\S 5$, under $(\beta)$ of Remark 5 , we have $\eta_{k}(t) \geqq \lambda_{k}(t)=-\psi(t)-$ $\gamma\left|\xi_{k}(t)\right|$; then, as we have noticed there, there is a subsequence $\left[\lambda_{k_{s}}\right]$ which converges weakly in $L_{1}(G)$ toward some $L$-integrable function $-\psi(t)-\gamma \sigma(t)$, $\sigma(t) \geqq 0, t \in G$. On the other hand, in the proof of (7.i), we have $-\psi(t)-\gamma|\xi(t)| \leqq$ $\eta(t)=f_{0}(t, x(t), u(t)) \leqq \bar{\eta}(t), t \in G$, where $\psi,|\xi|, \bar{\eta} \in L_{1}(G)$, and hence $\eta \in L_{1}(G)$.

$\left(\gamma_{0}\right)$ We have $x, x_{k} \in\left(L_{p}(G)\right)^{n},\left\|x_{k}-x\right\|_{p} \rightarrow 0, \xi, \xi_{k} \in\left(L_{1}(G)\right)^{r}, \xi_{k} \rightarrow \xi$ weakly in $\left(L_{1}(G)\right)^{r}$, for some $p, 1 \leqq p<\infty$, and there exists a real valued function $\psi(t) \geqq 0$, $t \in G, \psi \in L_{1}(G)$, and constants $\gamma, \gamma^{\prime} \geqq 0$ such that $f_{0}(t, x, u) \geqq-\psi(t)-\gamma|x|^{p}-$ $\gamma^{\prime}|f(t, x, u)|$ for all $(t, x, u) \in M$.

Indeed, as in $\S 5$, under $(\gamma)$ of Remark 5 , we have $\eta_{k}(t) \geqq \lambda_{k}(t)=-\psi(t)-$ $\gamma\left|x_{k}(t)\right|-\gamma^{\prime}\left|\xi_{k}(t)\right|$; also we noticed there that there is a subsequence $\left[\lambda_{k s}\right]$ which converges weakly in $L_{1}(G)$ toward some $L$-integrable function $-\psi(t)-\gamma|x(t)|-$ $\gamma^{\prime} \sigma(t), \sigma(t) \geqq 0, t \in G$. On the other hand, in the proof of (7.i), we have $-\psi(t)-$ $\gamma|x(t)|-\gamma^{\prime}|\xi(t)| \leqq \eta(t)=f_{0}(t, x(t), u(t)) \leqq \bar{\eta}(t), t \in G$, and again $\eta \in L_{1}(G)$.

$\left(\delta_{0}\right)$ We have $x_{k} \in\left(L_{\infty}(G)\right)^{n},\left\|x_{k}\right\|_{\infty} \leqq L_{1}, \xi_{k} \in\left(L_{\infty}(G)\right)^{r},\left\|\xi_{k}\right\|_{\infty} \leqq L_{2}$ for given constants $L_{1}, L_{2}$, and there exists a real valued function $\psi(t) \geqq 0, t \in G, \psi \in L_{1}(G)$, and a real valued monotone nondecreasing function $\sigma(\xi) \geqq 0,0 \leqq \xi<\infty$, such that $f_{0}(t, x, u) \geqq-\psi(t) \sigma(|x|+|f(t, x, u)|)$ for all $(t, x, u) \in M$.

Other analogous cases can be treated similarly.

Remark 12. Statement (7.i) is usually applied to sequences for which it is known that

$$
i=\varliminf_{k \rightarrow \infty} \int_{G} \eta_{k}(t) d t<\infty
$$

The complementary inequality $i>-\infty$ required in statement (7.i) is certainly satisfied under any one of the conditions $\left(\alpha_{0}\right),\left(\beta_{0}\right),\left(\gamma_{0}\right),\left(\delta_{0}\right)$ stated in Remark 11. These cases reduce to the cases $(\alpha-\delta)$ of Remark 5 .

Remark 13. Solely under the hypotheses of statement (7.i), the function $\eta(t)=f(t, x(t), u(t)), t \in G$, may not be $L$-integrable in $G$. This can be seen in the two examples below.

Example 1. Take $v=n=m=r=1, \quad U=E_{1}, \quad A=[0,1] \times E_{1}, \quad M=A \times E_{1}$, $f(t, x, u)=u$, and $f_{0}(t, x, u)=0$ for $t=0, f_{0}(t, x, u)=t^{-1}\left(u^{2}-1\right)$ for $0<t \leqq 1$. Condition $(C)$ is obviously satisfied. We take now $x_{k}(t)=t-i / k, u_{k}(t)=1$ for $i / k \leqq t \leqq i / k+1 / 2 k, \quad x_{k}(t)=(i+1) / k-t, \quad u_{k}(t)=-1 \quad$ for $i / k+1 / 2 k \leqq t \leqq(i+1) / k$, $k=1,2, \ldots$, so that $\eta_{k}(t)=f_{0}\left(t, x_{k}(t), u_{k}(t)\right)=0, \xi_{k}(t)=u_{k}(t), t \in G$. Here $x_{k}(t)$ converges uniformly in $[0,1]$ to $x(t)=0$, and $\xi_{k}(t)$ converges weakly in $L_{1}(G)$ to $\xi(t)=0$. Now we have $0=\xi(t)=f(t, x(t), u(t))$ only for $u(t)=0,0<t \leqq 1$, and finally, $\eta(t)=f_{0}(t, x(t), u(t))=-t^{-1}$ is not $L$-integrable in $[0,1]$. Note that $\xi_{k} \rightarrow \xi$ weakly, and $u_{k} \rightarrow u$ weakly in $L_{1}$ (as well as in any $L_{p}, p \geqq 1$ ). Finally, the sets $Q(t)$ are here the sets $\tilde{Q}(t)=\left[\left(z^{0}, z\right) \mid z^{0} \geqq t^{-1}\left(z^{2}-1\right)\right]$, certainly closed, convex, and satisfying property $(Q)$ for every $t \in(0,1]$. Note that, if $\bar{\eta}$ denotes the function of statement (5.i), then $\bar{\eta}$ is zero for $0 \leqq t \leqq 1$, and thus $\bar{\eta}$ is $L$-integrable, but $\eta$ is not. 
Example 2. Take $v=n=m=r=1, U=E_{1}, A=[0,1] \times E_{1}, f(t, x, u)=t u^{2}-1$, $f_{0}(t, x, u)=1-2(1+t) u^{2}+t(1+t) u^{4}$, so that both $f_{0}$ and $f$ are now continuous on $M=A \times E_{1}$. We take $x_{k}(t)=t-i / k, u_{k}(t)=2^{1 / 2} t^{-1 / 2}$ for $i / k \leqq t \leqq i / k+1 / 2 k$, $x_{k}(t)=(i+1) / k-t, u_{k}(t)=0$ for $i / k+1 / 2 k \leqq t \leqq(i+1) / k, i=1, \ldots, k-1, k=1,2, \ldots$, so that $\xi_{k}(t)=f\left(t, x_{k}(t), u_{k}(t)\right)=1$, or $=-1$, according as $t$ lies in one or the other set of intervals. Thus, $x_{k}(t)$ conveges uniformly in $[0,1]$ to $x(t)=0$, and $\xi_{k}(t)$ converges weakly in $L_{1}(G)$ to $\xi(t)=0$. Now we have $0=\xi(t)=f(t, x(t), u(t))$ only for $u(t)= \pm t^{-1 / 2}, 0<t \leqq 1$, and finally $\eta(t)=f_{0}(t, x(t), u(t))=-t^{-1}$ is not $L$-integrable in $[0,1]$. Note that the sets $\tilde{Q}(t)$ are here the sets $\tilde{Q}(t)=\left[\left(z^{0}, z\right) \mid z^{0} \geqq\right.$ $\left.1-s+s z^{2},-1 \leqq z<+\infty\right], s=1+t^{-1}$ certainly convex, closed, and satisfying property $(Q)$ for every $t \in(0,1]$. Also, note that $\eta_{k}(t)=f_{0}\left(t, x_{k}(t), u_{k}(t)\right)=1$ for all $t \in[0,1]$, (a.e.). The function $\bar{\eta}$ of statement (5.i) is here equal to 1 , and $\bar{\eta}$ is $L$-integrable while $\eta$ is not.

Remark 14. Let us consider the following condition $\left(\gamma_{0}\right)^{\prime}$, weaker than condition $\left(\gamma_{0}\right)$ of Remark 11 .

$\left(\gamma_{0}\right)^{\prime}$ We have $x_{k} \in\left(L_{p}(G)\right)^{n},\left\|x_{k}\right\|_{p} \leqq L_{1}, \xi_{k} \in\left(L_{q}(G)\right)^{r},\left\|\xi_{k}\right\|_{q} \leqq L_{2}$ for given constants $p, q, L_{1}, L_{2}, 1 \leqq p, q<\infty$, and there exists a real valued function $\psi(t) \geqq 0$, $t \in G, \psi \in L_{1}(G)$, and constants $\gamma, \gamma^{\prime} \geqq 0$ such that $f_{0}(t, x, u) \geqq-\psi(t)-\gamma|x|^{p}-$ $\gamma^{\prime}|f(t, x, u)|^{q}$ for all $(t, x, u) \in M$.

The condition $\left(y_{0}\right)^{\prime}$ certainly guarantees that the function $\eta$ in (7.i) is $L$-integrable. Indeed, in the proof of (7.i) we have $-\psi(t)-\gamma|x(t)|^{p}-\gamma^{\prime}|\xi(t)|^{q} \leqq \eta(t)=$ $f_{0}(t, x(t), u(t)) \leqq \bar{\eta}(t), t \in G$, where $\psi,|x|^{p},|\xi|^{q} \in L_{1}(G)$, and hence $\eta \in L_{1}(G)$. Nevertheless, from the condition $\left(\gamma_{0}\right)^{\prime}$ for $q>1$ we cannot derive functions $\lambda$, $\lambda_{k} \in L_{1}(G)$, such that $\eta_{k} \geqq \lambda_{k}, \lambda_{k} \rightarrow \lambda$ weakly in $L_{1}(G)$, as required in (7.4) of (7.i). Indeed, for $q>1$, the conclusion of (7.i) may fail to be valid under the weak condition $\left(\gamma_{0}\right)^{\prime}$ for $q>1$ without requirement (7.4), and this may happen even if we assume as in $\left(\gamma_{0}\right)$ that $\left\|x_{k}-x\right\|_{p} \rightarrow 0$ and, of course, $\xi_{k} \rightarrow \xi$ weakly in $\left(L_{q}(G)\right)^{r}$. This is shown by the following example for $q=2$.

Example. Take $v=n=m=r=1, G=[t \mid 0 \leqq t \leqq 1], A(t)=[x \mid 0 \leqq x \leqq 1], A=$ $G \times[0,1], \quad U=\{0\}, f(t, x, 0)=\left(-f_{0}\right)^{1 / 2}, f_{0}(t, x, 0)=0$ for $0 \leqq t \leqq 1,0 \leqq x \leqq 1$, $t+x \leqq 1 ; f_{0}(t, x, 0)=2 x^{-2}(1-t-x)$ for $0 \leqq t<1,0 \leqq x \leqq 1, t+x \geqq 1 ; f_{0}(1, x, 0)=0$ for $0 \leqq x \leqq 1$. Obviously, $f$ and $f_{0}$ satisfy property $(C)$, the sets $\tilde{Q}(t, x)$ are all half-straight lines, namely, $\tilde{Q}(t, x)=\left[\left(z^{0}, z\right) \mid z^{0} \geqq 0, z=0\right]$ in the first instance, $\tilde{Q}(t, x)=\left[\left(z^{0}, z\right) \mid z^{0} \geqq-z^{2}, z=\left(-2 x^{-2}(1-t-x)\right)^{1 / 2}\right]$ in the second instance, and $\tilde{Q}(t, x)=\left[\left(z^{0}, z\right) \mid z^{0} \geqq 0, z=0\right]$ in the third. Obviously, these sets have property $(Q)$ with respect to $x$ for all $t$. We take $x_{k}(t)=k^{-1}, u_{k}(t)=0, \xi_{k}(t)=\left(-\eta_{k}(t)\right)^{1 / 2}$ for $0 \leqq t \leqq 1, \eta_{k}(t)=0$ for $0 \leqq t \leqq 1-k^{-1}, \eta_{k}(t)=2 k^{2}\left(1-t-k^{-1}\right)$ for $1-k^{-1} \leqq t \leqq 1$, $\int_{0}^{1} \eta_{k}(t) d t=-1$. Here we have $x(t)=0, \xi(t)=0, \eta(t)=0,\left\|x_{k}-x\right\|_{1}=k^{-1} \rightarrow 0$, $\left\|\xi_{k}-\xi\right\|_{1}=2^{3 / 2} 3^{-1} k^{-1 / 2} \rightarrow 0$ as $k \rightarrow \infty$. On the other hand, $\int_{0}^{1} \eta(t) d t=0$ and (7.5) does not hold. Note that here we have $\eta \in L_{1}(G)$, and $f_{0}=-f^{2}$; hence, condition $\left(\gamma_{0}\right)^{\prime}$ holds for $q=2$, yet the conclusion of (7.i) does not hold. We prove now that $\xi_{k} \rightarrow 0$ weakly in $L_{2}(G)$. Indeed,

$\xi_{k}(t)=0 \quad$ for $0 \leqq t \leqq 1-k^{-1}, \quad \xi_{k}(t)=\left(-2 k^{2}\left(1-t-k^{-1}\right)\right)^{1 / 2} \quad$ for $1-k^{-1} \leqq t \leqq 1$, 
and for any fixed function $\varphi(t), 0 \leqq t \leqq 1, \varphi \in L_{2}(G)$, we have

$$
\left|\int_{0}^{1} \xi_{k}(t) \varphi(t) d t\right| \leqq\left[\int_{-k^{-1}}^{1} \xi_{k}^{2} d t\right]^{1 / 2}\left[\int_{-k^{-1}}^{1} \varphi^{2} d t\right]^{1 / 2}=\left[\int_{-k^{-1}}^{1} \varphi^{2} d t\right]^{1 / 2}
$$

Since $\varphi^{2} \in L_{1}(G)$ is absolutely integrable, the last integral approaches zero as $k \rightarrow \infty$. Thus, $\xi_{k} \rightarrow 0$ weakly in $L_{2}(G)$.

(7.ii) Let the hypotheses of (7.i) hold, except that the sets $\tilde{Q}(t, x)$ are required to satisfy only property $(K)$ with respect to $x$ in $A_{G}-H_{0}$, and $\xi_{k}(t) \rightarrow \xi(t)$ pointwise a.e. in $G$ as $k \rightarrow \infty$. Then the conclusion of (7.i) holds.

The proof is the same as for (7.i), where use is made of (5.ii).

Requirement (7.4) and the $L$-integrability of $\eta$ in statement (7.ii) can be guaranteed under the same conditions, that is $\left(\alpha_{0}\right)$, or $\left(\beta_{0}\right)$ with $\gamma=0$, or $\left(\gamma_{0}\right)$ with $\gamma=0$, or $\left(\delta_{0}\right)$ of Remark 11. Conditions $\left(\beta_{0}\right)$ with $\gamma>0$, and $\left(\gamma_{0}\right)$ with $\gamma>0$ do not apply to statement (7.ii) (cfr. discussion in $\S 5$ ).

(7.iii) Let the hypotheses of (7.i) hold, except that the sets $\tilde{Q}(t, x)$ are only assumed to be convex and closed, and $\xi_{k} \rightarrow \xi$ weakly in $\left(L_{1}(G)\right)^{r}$ as $k \rightarrow \infty$, and $x_{k}(t)=x(t)$ for all $t$ and $k$. Then the conclusion of (7.i) holds.

The proof is the same as for (7.i), where use is made of (5.iii). Requirement (7.4) and the $L$-integrability of the function $\eta$ in statement (7.iii) can again be guaranteed under the same conditions, that is $\left(\alpha_{0}\right)$, or $\left(\beta_{0}\right)$ with $\gamma \geqq 0$, or $\left(\gamma_{0}\right)$ with $\gamma \geqq 0$, or $\left(\delta_{0}\right)$ of Remark 11 (exactly as for (7.i)).

Soley under the continuity condition $(C)$ for $f$ and $f_{0}$, and as in $\S 6$, if the sets $U(t)$ depend on $t$ only, and $\xi(t), x(t), \xi_{k}(t), \eta_{k}(t), x_{k}(t), u_{k}(t), t \in G, k=1,2, \ldots$, are measurable functions, $\xi, \xi_{k} \in\left(L_{1}(G)\right)^{r}, \eta_{k} \in L_{1}(G),\left(t, x_{k}(t)\right) \in A, u_{k}(t) \in U(t)$,

we may consider the differences

$$
\begin{gathered}
\xi_{k}(t)=f\left(t, x_{k}(t), u_{k}(t)\right), \quad \eta_{k}(t)=f_{0}\left(t, x_{k}(t), u_{k}(t)\right), \quad t \in G, \quad k=1,2, \ldots, \\
\varliminf_{G} \eta_{k}(t) d t=i,
\end{gathered}
$$

$$
\begin{aligned}
& \delta_{k}(t)=f\left(t, x_{k}(t), u_{k}(t)\right)-f\left(t, x(t), u_{k}(t)\right), \\
& \delta_{k}^{0}(t)=f_{0}\left(t, x_{k}(t), u_{k}(t)\right)-f_{0}\left(t, x(t), u_{k}(t)\right), \quad t \in G, \quad k=1,2, \ldots
\end{aligned}
$$

which exist because $U(t)$ depends on $t$ only. We shall assume as in (7.i) that $x_{k}(t) \rightarrow$ $x(t)$ pointwise a.e. in $G$, that $\xi_{k} \rightarrow \xi$ weakly in $\left(L_{1}(G)\right)^{r}$ as $k \rightarrow \infty$, and that $-\infty<$ $i<+\infty$. In addition let us assume here that

$$
\begin{gathered}
\delta_{k} \rightarrow 0 \quad \text { weakly in }\left(L_{1}(G)\right)^{r}, \\
\infty>\lim \int_{G} \delta_{k}^{0}(t) d t \geqq 0 .
\end{gathered}
$$

This occurs under natural conditions, as mentioned in Remark 9 (see also [3]). Actually, under such conditions $\delta_{k} \rightarrow 0$ strongly in $\left(L_{1}(G)\right)^{r}, \delta_{k}^{0} \rightarrow 0$ strongly in $L_{1}(G)$, and thus (7.6) holds trivially. Under assumption (7.6), then, for the analogous functions

$$
\begin{aligned}
& \bar{\xi}_{k}(t)=f\left(t, x(t), u_{k}(t)\right), \\
& \bar{\eta}_{k}(t)=f_{0}\left(t, x(t), u_{k}(t)\right), \quad t \in G, \quad k=1,2, \ldots,
\end{aligned}
$$


we have

$$
\bar{\zeta}_{k}(t)=\xi_{k}(t)-\delta_{k}(t), \quad \bar{\eta}_{k}(t)=\eta_{k}(t)-\delta_{k}^{0}(t), \quad t \in G, \quad k=1,2, \ldots
$$

Hence, $\bar{\xi}_{k} \rightarrow \xi$ weakly in $\left(L_{1}(G)\right)^{r}$,

$$
\varliminf \int_{G} \bar{\eta}_{k}(t) d t \leqq i,
$$

and statement (5.iii) applies. The following simple lower closure theorem, where reither property $(Q)$ nor property $(K)$ is required, is now a corollary of (5.iii).

(7.iv) Let the hypotheses of (7.i) hold, except that the sets $U(t)$ depend on $t$ only, the sets $\tilde{Q}(t, x)$ are only assumed to be convex and closed for every $(t, x) \in A_{G}-H_{0}$, and where $\xi, \xi_{k}, \delta_{k} \in\left(L_{1}(G)\right)^{r}, \eta_{k}, \delta_{k}^{0} \in L_{1}(G), \xi_{k} \rightarrow \xi, \delta_{k} \rightarrow 0$ weakly in $\left(L_{1}(G)\right)^{r}$, $\delta_{k}^{0} \rightarrow 0$ weakly in $L_{1}(G),-\infty<i=\underline{\lim } \int_{G} \eta_{k}(t) d t<+\infty$, and $x_{k}(t) \rightarrow x(t)$ pointwise a.e. in $G$. Then the conclusion of (7.i) holds.

We have seen that actually it is enough that (7.6) holds.

Remark 15. In the present situation, the lower closure theorem (7.i) essentially contains as a particular case (indeed, the particular case $\left(\alpha_{0}\right)$ under Remark 11 with $U$ a fixed set) an analogous proposition recently stated by M. F. BIDAUT (Quelques résultats d'existence pour des problèmes de contrôle optimal. C. R. Acad. Sci. Paris, t. 274, 1972, 62-65). As we have shown in $\$ 5$, moreover, these theorems can be formulated in the more general and more satisfactory context of orientor fields. - Added in proof: Two papers by L. D. BERKoviTz have been brought to my attention: Existence theorems in problems of optimal control. Studia Math. 44, 1972, 275-285; and Existence and lower closure theorems for abstract control problems. SIAM J. Control 12, 1974, 27-42. These papers deal with closure and lower closure theorems for weak convergence, under either property $(Q)$ with respect to $x$ only, or Lipschitz-type hypotheses. Essentially analogous comments (as above) hold for these papers. See also Remark 9 and the results in [3].

Remark 16. In applications (see, e.g., [1ij], [2]) we may have to consider a topological space $S$ of elements $y$, operators $L: S \rightarrow\left(L_{1}(G)\right)^{r}, M: S \rightarrow\left(L_{1}(G)\right)^{n}$, and a class $T$ of controls $u(t), t \in G$, as stated in Remark 9, and we may have to consider a functional $I[y, u]$, or $I: \Omega \rightarrow E_{1}$ defined in a suitable subset $\Omega$ of $S \times T$, of the form $I[y, u]=\int_{G} f_{0}(t,(M y)(t), u(t)) d t$. This may be called an abstract Lagrange problem. The requirements on the operators $L$ and $M$ have been mentioned in Remark 10. The lower closure theorems of the present $\$ 7$ apply, and essentially prove, under suitable closure properties of the class $\Omega$, that, as $y_{k} \rightarrow y$ in $S, u_{k} \in T,\left(y_{k}, u_{k}\right) \in \Omega$, there is some $u \in T$, such that $(y, u) \in \Omega$, and $I[y, u] \leqq$ $\varliminf_{i} I\left[y_{k}, u_{k}\right]$ as $k \rightarrow \infty$.

\section{Lower Semicontinuity Theorems}

We shall use essentially the notations of $\S 7$. Points in $E_{v}, E_{n}, E_{m}, E_{1}$ spaces will be denoted by $t=\left(t^{1}, \ldots, t^{\eta}\right), x=\left(x^{1}, \ldots, x^{n}\right), u=\left(u^{1}, \ldots, u^{m}\right)$, and $z^{0}$, or $\eta$, respectively. As usual, $A$ is a subset of $E_{v} \times E_{n}, A_{0}$ the projection of $A$ on $E_{v}$, and 
$A(t)=\left[x \in E_{n} \mid(t, x) \in A\right]$. For every $(t, x) \in A$ a subset $U(t, x)$ of $E_{m}$ is assigned, and $M$ denotes the set $[(t, x, u) \mid(t, x) \in A, u \in U(t, x)] \subset E_{v} \times E_{n} \times E_{m}$. Here $f_{0}(t, x, u)$ is a given real valued function on $M$. We denote as usual by $G$ and $T_{0}$ given subsets of $A_{0}$ in $E_{v}$; then $A_{G}$ and $H_{0}$ are the sets $A_{G}=A \cap\left(G \times E_{n}\right), H_{0}=A \cap\left(T_{0} \times E_{n}\right)$. Finally, we denote by $x(t)=\left(x^{1}, \ldots, x^{n}\right), u(t)=\left(u^{1}, \ldots, u^{m}\right), \eta(t), t \in G$, given functions on $G$.

In other words, we have the situation of $\S 7$ minus the function $f$. Namely, we are particularizing $\S 7$ by assuming $r=m, f(t, x, u)=u$. Thus, the sets $\tilde{Q}$ of $\S 7$ are here the sets

$$
Q_{0}(t, x)=\left[\left(z^{0}, u\right) \mid z^{0} \geqq f_{0}(t, x, u), u \in U(t, x)\right] .
$$

By assuming that $f_{0}$ satisfies condition $(C)$, we know that there is a set $T_{0}$ of measure zero such that the sets $A(t)$ are closed for all $t \in G-T_{0}$, the sets $M_{t}=$ $[(x, u) \mid(t, x, u) \in M]$ are also closed, and $f_{0}(t, x, u)$, as a function of $(x, u)$, is continuous on $M_{t}$. It is easy to see that, for any $t \in G-T_{0}$, the sets $Q_{0}(t, x)$ are closed and satisfy property $(K)$ with respect to $x$ in $A(t)$.

In $[1 \mathrm{~cd}]$ we expressed property $(Q)$ in terms of "seminormality" conditions. In the particular case under consideration $(r=m, f=u)$, and using the notations in [ $1 \mathrm{~cd}$ ], we say that for any given $t \in G-T_{0}$ the function $f_{0}(t, x, u)$, thought of as a function of $(x, u)$ in $M_{t}$, is seminormal with respect to $u$ at the point $(\bar{x}, \bar{u})$, $(\bar{x} \in A(t), \bar{u} \in U(t, \bar{x}))$, provided that given $\varepsilon>0$ there are real numbers $\delta>0$, and $r, b=\left(b_{1}, \ldots, b_{m}\right)$ such that

$$
\begin{gathered}
f_{0}(t, x, u) \geqq c+b u \quad \text { for all } x \in N_{\delta ; t}(\bar{x}) \text { and } u \in U(t, x) ; \\
f_{0}(t, \bar{x}, \bar{u})<c+b \bar{u}+\varepsilon .
\end{gathered}
$$

Here $b u$ denotes the usual inner product in $E_{m}$. Again, for any given $t \in G-T_{0}$, $f_{0}(t, x, u)$ is said to be seminormal with respect to $u$ in $A(t)$, provided $f_{0}(t, x, u)$ is seminormal with respect to $u$ at every point $(\bar{x}, \bar{u}), \bar{x} \in A(t), \bar{u} \in U(t, \bar{x})$.

The seminormality condition we have just defined is a slight extension of ToNELlI's and MCSHANE's seminormality condition for free problems of the calculus of variations.

For the particular sets $Q_{0}(t, x)$ defined above, our results in [1 cd] yield:

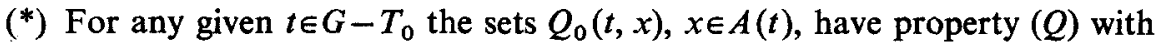
respect to $x$ in $A(t)$ if and only if $f_{0}(t, x, u)$, thought of as a function of $(x, u)$ in $M_{t}$, is "seminormal" with respect to $u$ for $x \in A(t)$.

The following lower semicontinuity theorems correspond to the lower closure statements (7.i-iii). Here $f=u$; hence, in the notations of $\S 7, \xi(t)=u(t), \xi_{k}(t)=$ $u_{k}(t)$.

(8.i) If $G$ is a measurable set of finite measure, and $T_{0}$ has measure zero, if $f_{0}(t, x, u)$ satisfies the continuity condition $(C)$, if the sets $U(t, x)$ are convex for every $(t, x) \in A_{G}-H_{0}$, if $f_{0}(t, x, u)$ is convex with respect to $u$ in $U(t, x)$, and the sets $Q_{0}(t, x)$ satisfy condition $(Q)$ with respect to $x$ in $A_{G}-H_{0}$ (or, equivalently, for every $t \in G-T_{0}, f_{0}(t, x, u)$ is seminormal with respect to $u$ for $\left.x \in A(t)\right)$, if $\eta_{k}(t), x(t), u(t), x_{k}(t), u_{k}(t), \lambda(t), \lambda_{k}(t), t \in G, k=1,2, \ldots$, are measurable functions with $x_{k}(t) \in A(t), \quad u_{k}(t) \in U\left(t, x_{k}(t)\right), \eta_{k}(t)=f_{0}\left(t, x_{k}(t), u_{k}(t)\right), t \in G$ (a.e.), if $u$, $u_{k} \in\left(L_{1}(G)\right)^{m}, \eta_{k} \in L_{1}(G), u_{k} \rightarrow u$ weakly in $\left(L_{1}(G)\right)^{m}, x_{k}(t) \rightarrow x(t)$ in measure, and 
$-\infty<i=\varliminf_{G} \eta_{k}(t) d t<\infty$, if $\eta_{k}(t) \geqq \lambda_{k}(t), \lambda, \lambda_{k} \in L_{1}(G), \lambda_{k} \rightarrow \lambda$ weakly in $L_{1}(G)$, then $x(t) \in A(t), u(t) \in U(t, x(t)), t \in G$ (a.e.), and if $\eta(t)=f_{0}(t, x(t), u(t)), t \in G$, then $\eta \in L^{-}(G)$, and $\int_{G} \eta(t) d t \leqq i$.

As in Remark 11 of $\S 7$, under either of the conditions $\left(\alpha_{0}\right),\left(\beta_{0}\right)$ there are functions $\lambda, \lambda_{k}$ satisfying the requirements above with $\eta \in L_{1}(G)$.

(8.ii) Let the hypotheses of (8.i) hold, except that $f_{0}(t, x, u)$ anly satisfies property $(C)$ and is convex with respect to $u$ for all $(t, x) \in A_{G}-H_{0}$ (no property $(Q)$ or seminormality required), and suppose $\eta_{k} \in L_{1}(G), u_{k}(t) \rightarrow u(t), x_{k}(t) \rightarrow x(t)$ pointwise in a.e. in $G$ as $k \rightarrow \infty$, and $-\infty<i<\infty$. Then the conclusion of (8.i) also holds.

In statement (8.ii) we can again determine functions $\lambda, \lambda_{k}$ satisfying the corresponding requirements in (8.i), so as to guarantee that $\eta \in L_{1}(G)$, under either condition $\left(\alpha_{0}\right)$, or condition $\left(\beta_{0}\right)$ with $\gamma=0$ of Remark 11 .

(8.iii) Let the hypotheses of (8.i) hold, except that $f_{0}(t, x, u)$ is only assumed to be convex with respect to $u$ for all $(t, x) \in A_{G}-H_{0}$ (no seminormality required), and $\eta_{k} \in L_{1}(G), u, u_{k} \in\left(L_{1}(G)\right)^{m}, u_{k} \rightarrow u$ weakly in $\left(L_{1}(G)\right)^{m}$, and $x_{k}(t)=x(t)$ for all $t$ and $k$.

In statement (8.iii) we can again determine functions $\lambda, \lambda_{k}$ satisfying the corresponding requirements in (8.i), so as to guarantee that $\eta \in L_{1}(G)$, under either condition $\left(\alpha_{0}\right)$, or condition $\left(\beta_{0}\right)$ with $\gamma \geqq 0$ of Remark 11 (exactly as for (8.i)).

We assume now that the sets $U(t)$ depend on $t$ only, and we consider the differences

$$
\delta_{k}^{0}(t)=f_{0}\left(t, x_{k}(t), u_{k}(t)\right)-f_{0}\left(t, x(t), u_{k}(t)\right), \quad t \in G, \quad k=1,2, \ldots,
$$

(8.iv) Let the hypotheses of (8.i) be satisfied, except that the sets $U(t)$ depend on $t$ only and are convex, $f_{0}(t, x, u)$ only satisfies property $(C)$ and is convex with respect to $u$ in $U(t)$ for every $(t, x) \in A_{G}-H_{0}$ (no property $(Q)$ or seminormality required), and $\eta_{k} \in L_{1}(G), u_{k} \rightarrow u$ weakly in $\left(L_{1}(G)\right)^{m}, \delta_{k}^{0} \rightarrow 0$ weakly in $L_{1}(G)$, $x_{k}(t) \rightarrow x(t)$ pointwise a.e. in $G$ as $k \rightarrow \infty$, and $-\infty<i<\infty$. Then the conclusion of (8.i) holds.

These statements (8.i-iii) are corollaries of the corresponding statements (7.i-iii).

Remark 17. If $S, T, L, M$ are as in Remarks 10 and 16 , then we may consider abstract free problems with the functional $I[y]$ of the form

$$
I[y]=\int_{G} f_{0}(t,(M y)(t),(L y)(t)) d t,
$$

or equivalently $I[y, u]=\int_{G} f_{0}(t,(M y)(t), u(t)) d t$, where $y$ and $u$ are related by the equations $(L y)(t)=u(t), t \in G$ (a.e.) (and the usual constraint relations $(M y)(t) \in$ $A(t), u(t) \in U(t,(M y)(t)), t \in G$ (a.e.) are satisfied). The lower semicontinuity theorems of the present section then apply to these problems.

Added in proof: By consistent use of the method and results of the present paper, and under suitable conditions of geometric character, we have further 
reduced, or eliminated, the requirements concerning property $(Q)$ in closure, lower closure, and semicontinuity theorems, in this paper: cf. L. CESARI, Lower semicontinuity and lower closure theorems without seminormality conditions. Annali Matem. pura appl. 98, 1974, 381-397.

This research was partially supported by AFOSR Research Project 71-2122 at the University of Michigan.

\section{References}

1. Cesari, L., (a) Existence theorems for weak and usual solutions in Lagrange problems with unilateral constraints. I and II. Trans. Amer. Math. Soc. 124, 369-412, 413-429 (1966). (b) Existence theorems for optimal controls of the Mayer type. SIAM J. Control 6, 517-552 (1968). (c) Seminormality and upper semicontinuity in optimal control. J. Optimization Theory Appl. 6, 114-137 (1970). (d) Lagrange problems of optimal control and convex sets not containing any straight line. Rend. Mat. Sem. Univ. Modena. (To appear.) (e) Closure, lower closure, and semicontinuity theorems in optimal control. SIAM J. Control 9, 287-315 (1971). (f) Existence theorems for multidimensional problems of optimal control. Differential Equations and Dynamic Systems. Academic Press 1967, 115-132. (g) Existence theorems for multidimensional Lagrange problems. J. Optimization Theory Appl. 1, 87-112 (1967). (h) Multidimensional Lagrange problems of optimization in a fixed domain and an application to a problem of magnetohydrodynamics. Arch. Rational Mech. Anal. 29, 81-104 (1968). (i) Existence theorems for abstract multidimensional control problems. J. Optimization Theory Appl. 6, 210-236 (1970). (j) Existence theorems for problems of optimization with distributed and boundary controls. Proc. Intern. Congress Math. Vol. 3, 157-161. Nice 1970.

2. Cesari, L., \& D. E. Cowles, Existence theorems in multidimensional problems of optimization with distributed and boundary' controls. Arch. Rational Mech. Anal. 46, 321-355 (1972).

3. Cesari, L., \& M. B. Suryanarayana, Closure theorems without seminormality conditions. Journ. Optimization Theory Appl. (To appear.)

4. CowLES, D. E., Upper semicontinuity properties of variable sets in optimal control. Journ. Optimization Theory Appl. 10, 222-236 (1972).

5. DAY, M., Normed Linear Spaces. New York-Heidelberg-Berlin: Springer 1973.

6. DUNFORD, N., \& B. J. PETTIS, Linear operators on summable functions. Trans. Amer. Math. Soc. 47, 323-392 (1940).

7. Edwards, R. E., Functional Analysis. New York: Holt, Rinehart, and Winston 1965.

8. KAISER, P., Weak forms of seminormality condition in problems of optimization. (To appear.)

9. MAZUR, S., Über konvexe Mengen in linearen normierten Räumen. Studia Math. 4, 70-84 (1933).

10. McShane, E. J., \& R. B. Warfield, On Filippov's implicit function lemma. Proc. Amer. Math. Soc. 18, 41-47 (1967).

11. YosidA, K., Functional Analysis, New York-Heidelberg-Berlin: Springer 1971.

Department of Mathematics

University of Michigan

Ann Arbor

(Received November 15, 1972) 